JOURNAL OF

FUNCTION SPACES AND APPLICATIONS

Volume 6, Number 1 (2008), 17-46
(C) 2008, Scientific Horizon http://www.jfsa.net

\title{
On dilation operators and sampling numbers
}

\author{
Jan Vybíral
}

(Communicated by Hans Triebel)

2000 Mathematics Subject Classification. 41A25, 41A46, $46 \mathrm{E} 35$.

Keywords and phrases. Linear and nonlinear approximation methods, Besov spaces, dilation operators, sampling operators.

Abstract. We consider the dilation operators $T_{k}: f \rightarrow f\left(2^{k} \cdot\right)$ in the frame of Besov spaces $B_{p q}^{s}\left(\mathbb{R}^{d}\right)$ with $1 \leq p, q \leq \infty$. If $s>0, T_{k}$ is a bounded linear operator from $B_{p q}^{s}\left(\mathbb{R}^{d}\right)$ into itself and there are optimal bounds for its norm, see $[4,2.3 .1]$. We study the situation in the case $s=0$, an open problem mentioned also in [4]. It turns out, that new effects based on Littlewood-Paley theory appear.

In the second part of the paper, we apply these results to the study of the so-called sampling numbers of the embedding

$$
i d: B_{p q_{1}}^{s_{1}}(\Omega) \rightarrow B_{p q_{2}}^{0}(\Omega),
$$

where $\Omega=(0,1)^{d}$. It was observed already in [13] that the estimates from above for the norm of the dilation operator have their immediate counterpart in the estimates from above for the sampling numbers. In this paper we show that even in the limiting case $s_{2}=0$ (left open so far), this general method supplies optimal results. 


\section{Introduction}

This paper is divided into two parts. In the first part, we consider the dilation operators

$$
T_{k}: f \rightarrow f\left(2^{k} \cdot\right), \quad k \in \mathbb{N},
$$

in the framework of Besov spaces $B_{p q}^{s}\left(\mathbb{R}^{d}\right)$. Their behaviour is well known if $1 \leq p, q \leq \infty$ and $s>0$, cf. [4, 2.3.1]. As mentioned there, the case $s=0$ remained open. Some partial results can be found in [1]. For $1 \leq p, q \leq \infty$ we supply the final answer to this problem showing that

$$
\left\|T_{k} \mid \mathcal{L}\left(B_{p q}^{0}\left(\mathbb{R}^{d}\right)\right)\right\| \approx 2^{-k \frac{d}{p}} \cdot \begin{cases}k^{\frac{1}{q}-\frac{1}{p}}, & \text { if } 1<p<\infty \text { and } p \geq \max (q, 2), \\ k^{\frac{1}{q}-\frac{1}{2}}, & \text { if } 1<p<\infty \text { and } 2 \geq \max (p, q), \\ 1, & \text { if } 1<p<\infty \text { and } q \geq \max (p, 2), \\ k^{\frac{1}{q}}, & \text { if } p=1 \text { or } p=\infty\end{cases}
$$

where $\left\|T_{k} \mid \mathcal{L}\left(B_{p q}^{0}\left(\mathbb{R}^{d}\right)\right)\right\|$ denotes the norm of the operator $T_{k}$ from $B_{p q}^{0}\left(\mathbb{R}^{d}\right)$ into itself. One observes, that for $1<p<\infty$ the number 2 plays an exceptional role. This effect has its origin in the Littlewood-Paley decomposition theorem.

The second part of the paper deals with applications to estimates of sampling numbers. Let us briefly sketch this approach.

Let $\Omega=(0,1)^{d}$ and let $B_{p q}^{s}(\Omega)$ denote the Besov spaces on $\Omega$, see Definition 2.7 for details. We try to approximate $f \in B_{p_{1} q_{1}}^{s_{1}}(\Omega)$ in the norm of another Besov space, say $B_{p_{2} q_{2}}^{s_{2}}(\Omega)$, by a linear sampling method

$$
S_{n} f=\sum_{j=1}^{n} f\left(x_{j}\right) h_{j},
$$

where $h_{j} \in B_{p_{2} q_{2}}^{s_{2}}(\Omega)$ and $x_{j} \in \Omega$. To give a meaning to the pointwise evaluation in (1.2), we suppose that

$$
s_{1}>\frac{d}{p_{1}} .
$$

Then the embedding $B_{p_{1} q_{1}}^{s_{1}}(\Omega) \hookrightarrow C(\bar{\Omega})$ holds true and the pointwise evaluation represents a bounded operator. Second, we always assume that the embedding $B_{p_{1} q_{1}}^{s_{1}}(\Omega) \hookrightarrow B_{p_{2} q_{2}}^{s_{2}}(\Omega)$ is compact. This is true if, and only if,

$$
s_{1}-s_{2}>d\left(\frac{1}{p_{1}}-\frac{1}{p_{2}}\right)_{+} .
$$


Concerning the parameters $p_{1}, p_{2}, q_{1}, q_{2}$ we always assume that they belong to $[1, \infty]$.

We measure the worst case error of $S_{n} f$ on the unit ball of $B_{p_{1} q_{1}}^{s_{1}}(\Omega)$, given by

$$
\sup \left\{\left\|f-S_{n} f\left|B_{p_{2} q_{2}}^{s_{2}}(\Omega)\|:\| f\right| B_{p_{1} q_{1}}^{s_{1}}(\Omega)\right\| \leq 1\right\} .
$$

The same worst case error may be considered also for nonlinear sampling methods

$$
S_{n} f=\varphi\left(f\left(x_{1}\right), \ldots, f\left(x_{n}\right)\right),
$$

where $\varphi: \mathbb{C}^{n} \rightarrow B_{p_{2} q_{2}}^{s_{2}}(\Omega)$ is an arbitrary mapping. We shall discuss the decay of (1.3) for linear (1.2) and nonlinear (1.4) sampling methods.

The case $s_{2} \neq 0$ was considered in [13], but the interesting limiting case $s_{2}=0$ was left open so far. It is the aim of this paper to close this gap. It was already pointed out in [13], see especially (2.6) in [13] for details, that the estimates from above for the dilation operators $T_{k}$ on the target space $B_{p_{2} q_{2}}^{s_{2}}\left(\mathbb{R}^{d}\right)$ have their direct counterparts in estimates from above for the decay of sampling numbers. Using this method, which will not be repeated here, a direct application of (1.1) supplies the estimates

$$
g_{n}^{\operatorname{lin}}(i d) \lesssim n^{-\frac{s}{d}} \cdot \begin{cases}(\log n)^{\frac{1}{q_{2}}-\frac{1}{p}}, & \text { if } 1<p<\infty \text { and } p \geq \max \left(q_{2}, 2\right) \\ (\log n)^{\frac{1}{q_{2}}-\frac{1}{2}}, & \text { if } 1<p<\infty \text { and } 2 \geq \max \left(p, q_{2}\right) \\ 1, & \text { if } 1<p<\infty \text { and } q_{2} \geq \max (p, 2) \\ (\log n)^{\frac{1}{q_{2}}}, & \text { if } p=1 \text { or } p=\infty\end{cases}
$$

where $g_{n}^{\operatorname{lin}}(i d)$ with $2 \leq n \in \mathbb{N}$ are the linear sampling numbers of the embedding

$$
i d: B_{p q_{1}}^{s}(\Omega) \rightarrow B_{p q_{2}}^{0}(\Omega), \quad s>\frac{d}{p} .
$$

Surprisingly, all estimates in (1.5) are sharp.

All the unimportant constants are denoted by the letter $c$, whose meaning may differ from one occurrence to another. If $\left\{a_{n}\right\}_{n=1}^{\infty}$ and $\left\{b_{n}\right\}_{n=1}^{\infty}$ are two sequences of positive real numbers, we write $a_{n} \lesssim b_{n}$ if, and only if, there is a positive real number $c>0$ such that $a_{n} \leq c b_{n}, n \in \mathbb{N}$. Furthermore, $a_{n} \approx b_{n}$ means that $a_{n} \lesssim b_{n}$ and simultaneously $b_{n} \lesssim a_{n}$.

We also discuss the case when $p_{1} \neq p_{2}$ and state some open problems connected to this question.

I would like to thank Winfried Sickel and Hans Triebel for many valuable discussions and comments on the topic. 


\section{Notation and definitions}

2.1 Besov spaces on $\mathbb{R}^{d}$. We use standard notation: $\mathbb{N}$ denotes the collection of all natural numbers, $\mathbb{Z}$ is the set of all integer numbers, $\mathbb{R}^{d}$ is Euclidean $d$-dimensional space, where $d \in \mathbb{N}$, and $\mathbb{C}$ stands for the complex plane. Let $S\left(\mathbb{R}^{d}\right)$ be the Schwartz space of all complex-valued rapidly decreasing, infinitely differentiable functions on $\mathbb{R}^{d}$ and let $S^{\prime}\left(\mathbb{R}^{d}\right)$ be its dual - the space of all tempered distributions.

Furthermore, $L_{p}\left(\mathbb{R}^{d}\right)$ with $1 \leq p \leq \infty$, are the standard Lebesgue spaces endowed with the norm

$$
\left\|f \mid L_{p}\left(\mathbb{R}^{d}\right)\right\|= \begin{cases}\left(\int_{\mathbb{R}^{d}}|f(x)|^{p} \mathrm{~d} x\right)^{1 / p}, & 1 \leq p<\infty \\ \underset{\substack{\operatorname{ess} \sup ^{d} \\ x \in \mathbb{R}^{d}}}{(x) \mid,} & p=\infty\end{cases}
$$

For $\psi \in S\left(\mathbb{R}^{d}\right)$ we denote by

$$
\widehat{\psi}(\xi)=(F \psi)(\xi)=(2 \pi)^{-d / 2} \int_{\mathbb{R}^{d}} e^{-i<x, \xi>} \psi(x) \mathrm{d} x, \quad x \in \mathbb{R}^{d},
$$

its Fourier transform and by $\psi^{\vee}$ or $F^{-1} \psi$ its inverse Fourier transform. With the aid of duality, they are extended to $S^{\prime}\left(\mathbb{R}^{d}\right)$.

We give a Fourier-analytic definition of the Besov spaces, which relies on the so-called dyadic resolution of unity. Let $\varphi \in S\left(\mathbb{R}^{d}\right)$ with

$$
\varphi(x)=1 \quad \text { if } \quad|x| \leq 1 \quad \text { and } \quad \varphi(x)=0 \quad \text { if } \quad|x| \geq \frac{3}{2} .
$$

We put $\varphi_{0}=\varphi$ and $\varphi_{j}(x)=\varphi\left(2^{-j} x\right)-\varphi\left(2^{-j+1} x\right)$ for $j \in \mathbb{N}$ and $x \in \mathbb{R}^{d}$. This leads to the identity

$$
\sum_{j=0}^{\infty} \varphi_{j}(x)=1, \quad x \in \mathbb{R}^{d}
$$

Definition 2.1. Let $s \in \mathbb{R}, 1 \leq p, q \leq \infty$. Then $B_{p q}^{s}\left(\mathbb{R}^{d}\right)$ is the collection of all $f \in S^{\prime}\left(\mathbb{R}^{d}\right)$ such that

$$
\left\|f \mid B_{p q}^{s}\left(\mathbb{R}^{d}\right)\right\|=\left(\sum_{j=0}^{\infty} 2^{j s q}\left\|\left(\varphi_{j} \widehat{f}\right)^{\vee} \mid L_{p}\left(\mathbb{R}^{d}\right)\right\|^{q}\right)^{1 / q}<\infty
$$

(with the usual modification for $q=\infty$ ).

Remark 2.2. These spaces have a long history. In this context we recommend [7], [10], [11] and [12] as standard references. Let us mention 
that the spaces $B_{p q}^{s}\left(\mathbb{R}^{d}\right)$ are independent of the choice of $\varphi$ in the sense of equivalent norms.

2.2. Local means and atomic decompositions. We use the characterisation of Besov spaces by local means. We refer to [2], [3] and [12] for further details. Let us sketch this approach.

Let $B=\left\{y \in \mathbb{R}^{d}:|y|<1\right\}$ be the unit ball in $\mathbb{R}^{d}$ and let $\kappa$ be a $C^{\infty}$ function in $\mathbb{R}^{d}$ with $\operatorname{supp} \kappa \subset B$,

$$
\kappa^{\vee}(\xi) \neq 0 \quad \text { if } \quad 0<|\xi|<\epsilon \quad \text { and } \quad\left(D^{\alpha} \kappa^{\vee}\right)(0)=0 \quad \text { if } \quad|\alpha| \leq s .
$$

for some $\epsilon>0$. Furthermore, let $\kappa_{0}$ be a second $C^{\infty}$ function with $\operatorname{supp} \kappa_{0} \subset B$ and $\kappa_{0}^{\vee}(0) \neq 0$.

Then

$$
\begin{gathered}
\left\|f\left|B_{p q}^{s}\left(\mathbb{R}^{d}\right)\|\approx\| \mathcal{K}_{0}(1, f)\right| L_{p}\left(\mathbb{R}^{d}\right)\right\|+\left(\sum_{j=1}^{\infty} 2^{j s q}|| \mathcal{K}\left(2^{-j}, f\right)\left|L_{p}\left(\mathbb{R}^{d}\right)\right|^{q}\right)^{1 / q}, \\
f \in S^{\prime}\left(\mathbb{R}^{d}\right),
\end{gathered}
$$

where

$$
\mathcal{K}(t, f)(x)=\int_{\mathbb{R}^{d}} \kappa(y) f(x+t y) d y=t^{-d} \int_{\mathbb{R}^{d}} \kappa\left(\frac{y-x}{t}\right) f(y) d y, \quad x \in \mathbb{R}^{d},
$$

appropriately interpreted for $f \in S^{\prime}\left(\mathbb{R}^{d}\right)$. The meaning of $\mathcal{K}_{0}(1, f)$ is defined in the same way with $\kappa_{0}$ instead of $\kappa$.

We shall need only one part of (2.4), namely the estimates from below of $\left\|f \mid B_{p q}^{s}\left(\mathbb{R}^{d}\right)\right\|$. In that case some of the assumptions may be omitted. The inspection of the proof of (2.4), see [8], shows that if $\kappa$ is a $C^{\infty}$ function in $\mathbb{R}^{d}$ with $\operatorname{supp} \kappa \subset B$ and $\kappa^{\vee}(0)=0$, then

$$
\left\|f \mid B_{p q}^{0}\left(\mathbb{R}^{d}\right)\right\| \gtrsim\left(\sum_{j=1}^{\infty}\left\|\mathcal{K}\left(2^{-j}, f\right) \mid L_{p}\left(\mathbb{R}^{d}\right)\right\|^{q}\right)^{1 / q},
$$

Secondly we rely on atomic decompositions. We refer again to [12] for details.

Recall that $\mathbb{Z}^{d}$ stands for the lattice of all points in $\mathbb{R}^{d}$ with integer-valued components. Furthermore, $Q_{\nu m}$ denotes the closed cube in $\mathbb{R}^{d}$ with sides parallel to the axes of coordinates, centred at $2^{-\nu} m$, and with side length $2^{-\nu}$ where $m \in \mathbb{Z}^{d}$ and $\nu \in \mathbb{N}_{0}$. If $Q$ is a cube in $\mathbb{R}^{d}$ and $c>0$ then $c Q$ is a cube in $\mathbb{R}^{d}$ concentric with $Q$ and with side length $c$ times of the side length of $Q$. 
Definition 2.3. Let $K \in \mathbb{N}_{0}, L \in \mathbb{N}_{0}, \nu \in \mathbb{N}_{0}, m \in \mathbb{Z}^{d}$ and $c \geq 1$. A $K$-times differentiable function $a(x)$ is called an $(K, L)$ atom centred on $Q_{\nu m}$ if

$$
\begin{gathered}
\operatorname{supp} a \subset c Q_{\nu m}, \\
\left|D^{\alpha} a(x)\right| \leq 2^{|\alpha| \nu}, \quad \text { for } \quad|\alpha| \leq K
\end{gathered}
$$

and

$$
\int_{\mathbb{R}^{d}} x^{\beta} a(x) d x=0, \quad \text { for } \quad|\beta|<L \quad \text { and } \quad \nu \geq 1 .
$$

Remark 2.4. We add a few comments on Definition 2.3. The number $K$ denotes the smoothness of the atom (see (2.7)), $L$ gives the number of vanishing moments, see (2.8), and the pair $(\nu, m)$ denotes the location of $\operatorname{supp} a$ (see (2.6)). Let us note that if $\nu=0$ or $L=0$, the condition (2.8) is empty and no moment conditions are required.

Theorem 2.5. Let $1 \leq p, q \leq \infty$ and $s \in \mathbb{R}$. Let $K \in \mathbb{N}_{0}, L \in \mathbb{N}_{0}$ with

$$
K>s \text { and } L>-s
$$

be fixed. Let $a_{\nu m}$ be $(K, L)$ atoms centred on $Q_{\nu m}$ and let

$$
\lambda=\left\{\lambda_{\nu m}: \nu \in \mathbb{N}_{0}, m \in \mathbb{Z}^{d}\right\}
$$

be a sequence of complex numbers with

$$
\left\|\lambda \mid b_{p q}^{s}\right\|=\left(\sum_{\nu=0}^{\infty} 2^{\nu\left(s-\frac{d}{p}\right) q}\left(\sum_{m \in \mathbb{Z}^{d}}\left|\lambda_{\nu m}\right|^{p}\right)^{\frac{q}{p}}\right)^{\frac{1}{q}}<\infty
$$

(appropriately modified if $p=\infty$ and/or $q=\infty$ ).

Then the series

$$
\sum_{\nu=0}^{\infty} \sum_{m \in \mathbb{Z}^{d}} \lambda_{\nu m} a_{\nu m}
$$

converges in $S^{\prime}\left(\mathbb{R}^{d}\right)$ to a distribution $f \in B_{p q}^{s}\left(\mathbb{R}^{d}\right)$ and

$$
\left\|f\left|B_{p q}^{s}\left(\mathbb{R}^{d}\right)\|\lesssim\| \lambda\right| b_{p q}^{s}\right\|
$$


Remark 2.6. We denote by $\chi_{\nu m}$ the characteristic function of $Q_{\nu m}$. Then

$$
\left\|\lambda \mid b_{p q}^{s}\right\|=\left(\sum_{\nu=0}^{\infty} 2^{s q}\left\|\sum_{m \in \mathbb{Z}^{d}} \lambda_{\nu m} \chi_{\nu m} \mid L_{p}\left(\mathbb{R}^{d}\right)\right\|^{q}\right)^{\frac{1}{q}},
$$

again appropriately modified if $q=\infty$.

2.3. Besov spaces on domains. Let $\Omega$ be a bounded domain. Let $D(\Omega)=C_{0}^{\infty}(\Omega)$ be the collection of all complex-valued infinitelydifferentiable functions with compact support in $\Omega$ and let $D^{\prime}(\Omega)$ be its dual - the space of all complex-valued distributions on $\Omega$.

Let $g \in S^{\prime}\left(\mathbb{R}^{d}\right)$. Then we denote by $g \mid \Omega$ its restriction to $\Omega$ :

$$
(g \mid \Omega) \in D^{\prime}(\Omega), \quad(g \mid \Omega)(\psi)=g(\psi) \quad \text { for } \quad \psi \in D(\Omega) .
$$

Definition 2.7. Let $\Omega$ be a bounded domain in $\mathbb{R}^{d}$. Let $s \in \mathbb{R}$, $1 \leq p, q \leq \infty$. Then

$$
B_{p q}^{s}(\Omega)=\left\{f \in D^{\prime}(\Omega): \exists g \in B_{p q}^{s}\left(\mathbb{R}^{d}\right): g \mid \Omega=f\right\}
$$

and

$$
\left\|f\left|B_{p q}^{s}(\Omega)\|=\inf \| g\right| B_{p q}^{s}\left(\mathbb{R}^{d}\right)\right\|,
$$

where the infimum is taken over all $g \in B_{p q}^{s}\left(\mathbb{R}^{d}\right)$ such that $g \mid \Omega=f$.

\section{Dilation operators}

Let $s \in \mathbb{R}, 1 \leq p, q \leq \infty$ and $k \in \mathbb{N}$. Then the dyadic dilation operator

$$
\left(T_{k} f\right)(x)=f\left(2^{k} x\right), \quad x \in \mathbb{R}^{d},
$$

is a bounded operator from $B_{p, q}^{s}\left(\mathbb{R}^{d}\right)$ into itself. Let us mention, that (3.1) has to be understood in the distributional sense. In this section we study the dependence of the norm of $T_{k}$ on $k$.

First, we recall known results.

Lemma 3.1. Let $s \geq 0,1 \leq p, q \leq \infty$ and $k \in \mathbb{N}$. Then the operator $T_{k}$ is bounded on $B_{p, q}^{s}\left(\mathbb{R}^{d}\right)$ and its norm is bounded by $c 2^{k\left(s-\frac{d}{p}\right)}$ if $s>0$ and by $c 2^{-k \frac{d}{p}} k^{1 / q}$ if $s=0$. The constant $c$ does not depend on $k \in \mathbb{N}$.

For the proof, we refer to $[1,1.7]$ and $[4,2.3 .1]$. If $s>0$, the estimate given by Lemma 3.1 is sharp (cf. [4]). But if $s=0$, the result can be improved. 
Proposition 3.2. Let $1<p<\infty, 1 \leq q \leq \infty, k \in \mathbb{N}$ and let $T_{k}$ be defined by (3.1). Then

$$
\left\|T_{k} \mid \mathcal{L}\left(B_{p q}^{0}\left(\mathbb{R}^{d}\right)\right)\right\| \leq c 2^{-k \frac{d}{p}} \cdot \begin{cases}k^{\frac{1}{q}-\frac{1}{p}}, & \text { if } p \geq \max (q, 2), \\ k^{\frac{1}{q}-\frac{1}{2}}, & \text { if } 2 \geq \max (p, q), \\ 1, & \text { if } q \geq \max (p, 2),\end{cases}
$$

for some $c$ which is independent of $k$.

Remark 3.3. The estimates covered by (3.2) may be summarised to

$$
\left\|T_{k} \mid \mathcal{L}\left(B_{p q}^{0}\left(\mathbb{R}^{d}\right)\right)\right\| \leq c 2^{-k \frac{d}{p}} \cdot k^{\frac{1}{q}-\frac{1}{\max (p, q, 2)}} .
$$

Proof. Elementary calculation involving only (2.1) shows that

$$
\left(\varphi_{j}(\xi) f\left(2^{k} \cdot\right)^{\curlyvee}(\xi)\right)^{\vee}(x)=2^{-k d}\left(\varphi_{j}(\xi) \widehat{f}\left(2^{-k} \xi\right)\right)^{\vee}(x)=\left(\varphi_{j}\left(2^{k} \xi\right) \widehat{f}(\xi)\right)^{\vee}\left(2^{k} x\right) .
$$

From (2.3) with $f\left(2^{k} x\right)$ in place of $f(x)$ we obtain

$$
\begin{aligned}
\left\|f\left(2^{k} \cdot\right) \mid B_{p, q}^{0}\left(\mathbb{R}^{d}\right)\right\| & =\left(\sum_{j=0}^{\infty}\left\|\left(\varphi_{j}\left(2^{k} \cdot\right) \widehat{f}\right)^{\vee}\left(2^{k} x\right) \mid L_{p}\left(\mathbb{R}^{d}\right)\right\|^{q}\right)^{1 / q} \\
& =2^{-k \frac{d}{p}}\left(\sum_{j=0}^{\infty}\left\|\left(\varphi_{j}\left(2^{k} \cdot\right) \widehat{f}\right)^{\vee} \mid L_{p}\left(\mathbb{R}^{d}\right)\right\|^{q}\right)^{1 / q}
\end{aligned}
$$

If $j \geq k+1$, then $\varphi_{j}\left(2^{k} x\right)=\varphi_{j-k}(x)$. This gives

$$
\begin{aligned}
2^{-k \frac{d}{p}}\left(\sum_{j=k+1}^{\infty}\left\|\left(\varphi_{j}\left(2^{k} \cdot\right) \widehat{f}\right)^{\vee} \mid L_{p}\left(\mathbb{R}^{d}\right)\right\|^{q}\right)^{1 / q} \\
=2^{-k \frac{d}{p}}\left(\sum_{j=1}^{\infty}\left\|\left(\varphi_{j} \widehat{f}\right)^{\vee} \mid L_{p}\left(\mathbb{R}^{d}\right)\right\|^{q}\right)^{1 / q} \\
\leq 2^{-k \frac{d}{p}}\left\|f \mid B_{p, q}^{0}\left(\mathbb{R}^{d}\right)\right\| .
\end{aligned}
$$

If $j=0$, we use (2.2) and Hausdorff-Young inequality

$$
\begin{aligned}
\left\|\left(\varphi_{0}\left(2^{k} \cdot\right) \widehat{f}\right)^{\vee} \mid L_{p}\left(\mathbb{R}^{d}\right)\right\| & =\left\|\left(\varphi_{0}\left(2^{k} \cdot\right) \varphi_{0} \widehat{f}\right)^{\vee} \mid L_{p}\left(\mathbb{R}^{d}\right)\right\| \\
& \approx\left\|\varphi_{0}\left(2^{k} \cdot\right)^{\vee} *\left(\varphi_{0} \widehat{f}\right)^{\vee} \mid L_{p}\left(\mathbb{R}^{d}\right)\right\| \\
& \leq\left\|\varphi_{0}\left(2^{k} \cdot\right)^{\vee}\left|L_{1}\left(\mathbb{R}^{d}\right)\|\cdot\|\left(\varphi_{0} \widehat{f}\right)^{\vee}\right| L_{p}\left(\mathbb{R}^{d}\right)\right\| \\
& \leq c\left\|f \mid B_{p, q}^{0}\left(\mathbb{R}^{d}\right)\right\| .
\end{aligned}
$$


In view of (3.4), (3.5) and (3.6), we have to prove that

$$
\left(\sum_{j=1}^{k}\left\|\left(\varphi_{j}\left(2^{k} \cdot\right) \widehat{f}\right)^{\vee} \mid L_{p}\left(\mathbb{R}^{d}\right)\right\|^{q}\right)^{1 / q} \leq c k^{\frac{1}{q}-\frac{1}{\max (p, q, 2)}}\left\|f \mid B_{p, q}^{0}\left(\mathbb{R}^{d}\right)\right\|
$$

with the constant $c$ independent of $k$ and $f$.

To prove (3.7), denote $\alpha=\max (p, q, 2)$. Using the Minkowski inequality and the Littlewood-Paley theorem one gets

$$
\begin{aligned}
& \left(\sum_{j=1}^{k}\left\|\left(\varphi_{j}\left(2^{k} \cdot\right) \widehat{f}\right)^{\vee} \mid L_{p}\left(\mathbb{R}^{d}\right)\right\|^{q}\right)^{1 / q} \\
& \quad \leq k^{\frac{1}{q}-\frac{1}{\alpha}}\left(\sum_{j=1}^{k}\left\|\left(\varphi_{j}\left(2^{k} \cdot\right) \widehat{f}\right)^{\vee} \mid L_{p}\left(\mathbb{R}^{d}\right)\right\|^{\alpha}\right)^{1 / \alpha} \\
& \quad \leq k^{\frac{1}{q}-\frac{1}{\alpha}}\left(\int_{\mathbb{R}^{d}}\left(\sum_{j=1}^{k}\left|\left(\varphi_{j}\left(2^{k} \cdot\right) \widehat{f}\right)^{\vee}(\xi)\right|^{\alpha}\right)^{p / \alpha} d \xi\right)^{1 / p} \\
& \quad \leq k^{\frac{1}{q}-\frac{1}{\alpha}}\left(\int_{\mathbb{R}^{d}}\left(\sum_{j=1}^{k}\left|\left(\varphi_{j}\left(2^{k} \cdot\right) \widehat{f}\right)^{\vee}(\xi)\right|^{2}\right)^{p / 2} d \xi\right)^{1 / p} \\
& \quad \leq c k^{\frac{1}{q}-\frac{1}{\alpha}}\left\|\left(\varphi_{0} \widehat{f}\right)^{\vee}\left|L_{p}\left(\mathbb{R}^{d}\right)\left\|\leq c k^{\frac{1}{q}-\frac{1}{\alpha}}\right\| f\right| B_{p, q}^{0}\left(\mathbb{R}^{d}\right)\right\| .
\end{aligned}
$$

Next, we prove that the estimates are sharp.

Theorem 3.4. Let $1 \leq p, q \leq \infty, k \in \mathbb{N}$ and let $T_{k}$ be defined by (3.1). Then

$$
\left\|T_{k} \mid \mathcal{L}\left(B_{p q}^{0}\left(\mathbb{R}^{d}\right)\right)\right\| \approx 2^{-k \frac{d}{p}} \cdot \begin{cases}k^{\frac{1}{q}-\frac{1}{p}}, & \text { if } 1<p<\infty \text { and } p \geq \max (q, 2), \\ k^{\frac{1}{q}-\frac{1}{2}}, & \text { if } 1<p<\infty \text { and } 2 \geq \max (p, q), \\ 1, & \text { if } 1<p<\infty \text { and } q \geq \max (p, 2), \\ k^{\frac{1}{q}}, & \text { if } p=1 \text { or } p=\infty,\end{cases}
$$

where the constants of equivalence do not depend on $k$.

Remark 3.5. Let us mention, that at $p=1$, there is a jump in the exponent of $k$ caused by the absence of the Littlewood-Paley assertion for $p=1$. At $p=\infty$, no such a jump appears.

Proof. In view of Lemma 3.1 and Proposition 3.2, we have to prove the estimates from below.

Step 1: $p=1$. 
Let $\psi \in S\left(\mathbb{R}^{d}\right)$ be a non-negative function with support in $\left\{x \in \mathbb{R}^{d}\right.$ : $|x| \leq 1 / 8\}$ and $\int_{\mathbb{R}^{d}} \psi(x) d x=1$. We show, that

$$
\left\|\psi\left(2^{k} \cdot\right) \mid B_{1, q}^{0}\left(\mathbb{R}^{d}\right)\right\| \gtrsim 2^{-k d} \cdot k^{\frac{1}{q}}, \quad k \in \mathbb{N},
$$

for $1 \leq q \leq \infty$.

We take a function $\kappa \in C^{\infty}(\mathbb{R})$ with

$$
\begin{gathered}
\operatorname{supp} \kappa \subset B=\left\{y \in \mathbb{R}^{d}:|y|<1\right\}, \quad \kappa^{\vee}(0)=0, \\
\kappa(x)=1 \text { if } x \in M=\left\{z \in \mathbb{R}^{d}:|z-(1 / 2,0, \ldots, 0)|<1 / 4\right\}
\end{gathered}
$$

and

$$
\kappa(x) \geq 0 \quad \text { if } \quad x_{1} \geq 0 .
$$

Simple calculation shows that if $j=1,2, \ldots, k$ and $\left|x-\left(\frac{1}{2} \cdot \frac{1}{2^{j}}, 0, \ldots, 0\right)\right|<$ $\frac{1}{2 j} \cdot \frac{1}{8}$ then

$$
\operatorname{supp}_{y} \psi\left(2^{k} x+2^{k-j} y\right) \subset M
$$

For these $x$ we get

$$
\begin{aligned}
\mathcal{K}\left(2^{-j}, \psi\left(2^{k} \cdot\right)\right)(x) & =\int_{\mathbb{R}^{d}} \kappa(y) \psi\left(2^{k} x+2^{k-j} y\right) d y \\
& =\int_{\mathbb{R}^{d}} \psi\left(2^{k} x+2^{k-j} y\right) d y=2^{(j-k) d} .
\end{aligned}
$$

Hence,

$$
\left\|\mathcal{K}\left(2^{-j}, \psi\left(2^{k} \cdot\right)\right) \mid L_{1}\left(\mathbb{R}^{d}\right)\right\| \gtrsim 2^{-j d} \cdot 2^{(j-k) d}=2^{-k d} .
$$

We insert (3.10) for $j=1,2, \ldots, k$ into (2.5). This completes the proof of (3.9).

Step 2: $p=\infty$.

We consider again a non-negative function $\psi \in S\left(\mathbb{R}^{d}\right)$ with $\operatorname{supp} \psi \subset$ $\left\{x \in \mathbb{R}^{d}:|x| \leq 1 / 8\right\}$ and $\int_{\mathbb{R}^{d}} \psi(x) d x=1$. Let

$$
\psi_{j}(x)=\sum_{\substack{0 \leq l_{i} \leq 2^{j-\gamma} \\ i=1,2, \ldots, d}} \psi\left(x-\left(2^{2 j}+l_{1}, l_{2}, \ldots, l_{d}\right)\right), \quad j \geq \gamma
$$

and

$$
f(x)=\sum_{j=\gamma}^{\infty} \psi_{j}(x), \quad x \in \mathbb{R}^{d},
$$

where the constant $\gamma \in \mathbb{N}$ will be chosen later on depending only on $d$. 
We observe, that (3.11) inserted into (3.12) represents an atomic decomposition of $f$ (see Theorem 2.5 for details) and, consequently, $f$ belongs to every space $B_{\infty, q}^{0}\left(\mathbb{R}^{d}\right), 1 \leq q \leq \infty$. We use again the local means to show that

$$
\left\|f\left(2^{k} \cdot\right) \mid B_{\infty, q}^{0}\left(\mathbb{R}^{d}\right)\right\| \geq c k^{\frac{1}{q}}
$$

with the constant $c$ independent of $k$.

Namely, we choose $\kappa$ as in Step 1, points

$$
x_{j}=\left(2^{k-2 j}-2^{-j-1}, 0, \ldots, 0\right), \quad j=\gamma, \ldots, k-\gamma,
$$

and show, that

$$
\mathcal{K}\left(2^{-j}, \psi_{k-j}\left(2^{k} \cdot\right)\right)\left(x_{j}\right) \geq 2^{-\gamma d}, \quad j=\gamma, \ldots, k-\gamma
$$

as well as

$$
\mathcal{K}\left(2^{-j}, \psi_{m}\left(2^{k} \cdot\right)\right)\left(x_{j}\right)=0, \quad m \neq k-j .
$$

From (3.14) and (3.15) it follows, that $\left\|\mathcal{K}\left(2^{-j}, f\left(2^{k} \cdot\right)\right) \mid L_{\infty}\left(\mathbb{R}^{d}\right)\right\| \geq 2^{-\gamma d}$, for all $j=\gamma, \ldots, k-\gamma$. Taking $q$-th power and summing up, we prove (3.13).

Let us first comment on (3.14).

$$
\begin{aligned}
\mathcal{K}\left(2^{-j}, \psi_{k-j}\left(2^{k} \cdot\right)\right)\left(x_{j}\right) & \\
\quad= & \sum_{\substack{0 \leq l_{i} \leq 2^{k-j-\gamma} \\
i=1,2, \ldots, d}} \int_{\mathbb{R}^{d}} \kappa(y) \psi\left(2^{k} x_{j}+2^{k-j} y-\left(2^{2(k-j)}+l_{1}, l_{2}, \ldots, l_{d}\right)\right) d y .
\end{aligned}
$$

It is a matter of simple calculation and triangle inequality that, if $2^{-\gamma} d^{1 / 2} \leq$ $\frac{1}{8}$, the following statement holds true: If the argument of $\psi$ in (3.16) lies in the support of $\psi$, then $\kappa(y)=1$.

Hence (3.16) is equal to

$$
2^{(k-j-\gamma) d} \int_{\mathbb{R}^{d}} \psi\left(2^{k-j} y\right) d y=2^{(k-j-\gamma) d} \cdot 2^{(j-k) d}=2^{-\gamma d} .
$$


To prove (3.15) we use an analog of (3.16)

$$
\begin{aligned}
\mathcal{K} & \left(2^{-j}, \psi_{m}\left(2^{k} \cdot\right)\right)\left(x_{j}\right) \\
& =\sum_{\substack{0 \leq l_{i} \leq 2^{m-\gamma} \\
i=1,2, \ldots, d}} \int_{\mathbb{R}^{d}} \kappa(y) \psi\left(2^{k} x_{j}+2^{k-j} y-\left(2^{2 m}+l_{1}, l_{2}, \ldots, l_{d}\right)\right) d y .
\end{aligned}
$$

It turns out that, for $m \neq k-j$ and any admissible $l$, there is no $y \in \mathbb{R}^{d}$ such that

$$
\left|2^{k} x_{j, 1}+2^{k-j} y_{1}-2^{2 m}+l_{1}\right| \leq \frac{1}{8} \quad \text { and } \quad\left|y_{1}\right| \leq 1 .
$$

Step 3. In this step, we shall prove the estimate

$$
\left\|T_{k} \mid \mathcal{L}\left(B_{p q}^{0}\left(\mathbb{R}^{d}\right)\right)\right\| \gtrsim 2^{-\frac{k d}{p}}, \quad k \in \mathbb{N},
$$

for $1<p<\infty$ and $1 \leq q \leq \infty$. Take any $f \in B_{p q}^{0}\left(\mathbb{R}^{d}\right)$ such that

$$
\alpha:=\left(\sum_{j=1}^{\infty}\left\|\mathcal{K}\left(2^{-j}, f\right) \mid L_{p}\left(\mathbb{R}^{d}\right)\right\|^{q}\right)^{1 / q}>0 .
$$

We use (2.5) and the simple formula

$$
\mathcal{K}\left(2^{-j}, f\left(2^{k} \cdot\right)\right)(x)=\mathcal{K}\left(2^{k-j}, f\right)\left(2^{k} x\right), \quad x \in \mathbb{R}^{d}, \quad j \geq k+1,
$$

and obtain

$$
\begin{aligned}
\left\|f\left(2^{k} \cdot\right) \mid B_{p q}^{0}\left(\mathbb{R}^{d}\right)\right\| & \gtrsim\left(\sum_{j=k+1}^{\infty}\left\|\mathcal{K}\left(2^{-j}, f\left(2^{k} \cdot\right)\right) \mid L_{p}\left(\mathbb{R}^{d}\right)\right\|^{q}\right)^{1 / q} \\
& =\left(\sum_{j=k+1}^{\infty}\left\|\mathcal{K}\left(2^{k-j}, f\right)\left(2^{k} \cdot\right) \mid L_{p}\left(\mathbb{R}^{d}\right)\right\|^{q}\right)^{1 / q} \\
& =2^{-\frac{k d}{p}} \alpha,
\end{aligned}
$$

which concludes the proof of (3.17).

Step 4.

Now we prove

$$
\left\|T_{k} \mid \mathcal{L}\left(B_{p q}^{0}\left(\mathbb{R}^{d}\right)\right)\right\| \gtrsim k^{\frac{1}{q}-\frac{1}{2}} 2^{-\frac{k d}{p}}, \quad k \in \mathbb{N},
$$

again for all $1<p<\infty$ and $1 \leq q \leq \infty$. 
First, we take a special decomposition of unity, see Definition 2.1. Namely, we suppose, that the function $\varphi$ satisfies

$$
\varphi(x)=1 \quad \text { if } \quad|x| \leq \frac{5}{4} \quad \text { and } \quad \varphi(x)=0 \quad \text { if } \quad|x| \geq \frac{3}{2} .
$$

It is easy to see, that

$$
\varphi_{j}(x)=\varphi\left(2^{-j} x\right)-\varphi\left(2^{-j+1} x\right)=1, \quad \text { if } \quad \frac{3}{4} \cdot 2^{j} \leq|x| \leq \frac{5}{4} \cdot 2^{j}, \quad j \in \mathbb{N} .
$$

Finally, we again take $\psi \in S\left(\mathbb{R}^{d}\right)$ with $\operatorname{supp} \psi \subset\left\{x \in \mathbb{R}^{d}:|x| \leq 1 / 8\right\}$. We define the functions $f_{k}$ through their Fourier transforms:

$$
\widehat{f_{k}}(\xi)=\sum_{j=1}^{k} \psi\left(2^{k}\left(\xi-\xi_{j}\right)\right), \quad \xi \in \mathbb{R}^{d}, \quad k \in \mathbb{N},
$$

where $\xi_{j}=\left(2^{-j}, 0, \ldots, 0\right)$. We shall show that

$$
\left\|f_{k} \mid B_{p q}^{0}\left(\mathbb{R}^{d}\right)\right\| \lesssim k^{\frac{1}{2}} 2^{k d\left(\frac{1}{p}-1\right)}, \quad k \in \mathbb{N}
$$

and

$$
\left\|f_{k}\left(2^{k} \cdot\right) \mid B_{p q}^{0}\left(\mathbb{R}^{d}\right)\right\| \gtrsim k^{\frac{1}{q}} 2^{-k d}, \quad k \in \mathbb{N} .
$$

First, we deal with (3.21). As the support of $\widehat{f}_{k}$ lies in the unit ball of $\mathbb{R}^{d}$, we may omit the terms with $j \geq 1$ in (2.3). Furthermore, since $1<p<\infty$ we may use the Littlewood-Paley decomposition theorem to estimate

$$
\begin{aligned}
\left\|f_{k} \mid B_{p q}^{0}\left(\mathbb{R}^{d}\right)\right\| & =\left\|\left(\varphi_{0} \widehat{f}_{k}\right)^{\vee} \mid L_{p}\left(\mathbb{R}^{d}\right)\right\| \\
& \approx\left\|\left(\sum_{j=1}^{\infty}\left|\left(\varphi_{1}\left(2^{j} \cdot\right) \varphi_{0} \widehat{f}_{k}\right)^{\vee}(x)\right|^{2}\right)^{1 / 2} \mid L_{p}\left(\mathbb{R}^{d}\right)\right\| \\
& =\left\|\left(\sum_{j=1}^{k}\left|\psi\left(2^{k}\left(\xi-\xi_{j}\right)\right)^{\vee}(x)\right|^{2}\right)^{1 / 2} \mid L_{p}\left(\mathbb{R}^{d}\right)\right\| \\
& =\left\|\left(\sum_{j=1}^{k}\left|2^{-k d} \psi^{\vee}\left(2^{-k} x\right) e^{i x \cdot \xi_{j}}\right|^{2}\right)^{1 / 2} \mid L_{p}\left(\mathbb{R}^{d}\right)\right\| \\
& =k^{\frac{1}{2}} 2^{-k d}\left\|\psi^{\vee}\left(2^{-k} x\right) \mid L_{p}\left(\mathbb{R}^{d}\right)\right\| \\
& =k^{\frac{1}{2}} 2^{k d\left(\frac{1}{p}-1\right)}\left\|\psi^{\vee} \mid L_{p}\left(\mathbb{R}^{d}\right)\right\| .
\end{aligned}
$$


To prove (3.22), observe that

$$
f_{k}\left(2^{k} \cdot \hat{)}(\xi)=2^{-k d} \sum_{j=1}^{k} \psi\left(\xi-2^{k} \xi_{j}\right), \quad \xi \in \mathbb{R}^{d}, \quad k \in \mathbb{N} .\right.
$$

Using again the support properties of $\psi$ and $\varphi_{j}$, we arrive at

$$
\begin{aligned}
\left\|f_{k}\left(2^{k} \cdot\right) \mid B_{p q}^{0}\left(\mathbb{R}^{d}\right)\right\| & \approx 2^{-k d}\left(\sum_{j=1}^{k}\left\|\psi\left(\cdot-2^{k} \xi_{j}\right)^{\vee} \mid L_{p}\left(\mathbb{R}^{d}\right)\right\|^{q}\right)^{1 / q} \\
& =k^{\frac{1}{q}} 2^{-k d}\left\|\psi^{\vee} \mid L_{p}\left(\mathbb{R}^{d}\right)\right\| .
\end{aligned}
$$

Step 5.

In this last step we prove the estimate

$$
\left\|T_{k} \mid \mathcal{L}\left(B_{p q}^{0}\left(\mathbb{R}^{d}\right)\right)\right\| \gtrsim k^{\frac{1}{q}-\frac{1}{p}} 2^{-\frac{k d}{p}}, \quad k \in \mathbb{N},
$$

again for all $1<p<\infty$ and $1 \leq q \leq \infty$.

Let $\psi \in S\left(\mathbb{R}^{d}\right)$ be a non-negative bump function with

$$
\operatorname{supp} \psi \subset[0,1]^{d} \text { and } \int_{\mathbb{R}^{d}} \psi(x) d x=1 .
$$

For a fixed $k \in \mathbb{N}$ we set

$$
\psi_{j}(x)=\sum_{l \in N_{j}^{k}} \psi(x-l), \quad x \in \mathbb{R}^{d}, j=1,2, \ldots, k-1,
$$

where

$N_{j}^{k}=\left\{l \in \mathbb{N}_{0}^{d}: 2^{j-1} \leq l_{1}-2^{j} \leq 2^{j}-1\right.$ and $0 \leq l_{i} \leq 2^{k}-1$ for $\left.i=2, \ldots, d\right\}$,

so that the set $N_{j}^{k}$ contains $2^{j-1+k(d-1)}$ vectors and $\psi_{j}$ consists of $2^{j-1+k(d-1)}$ copies of $\psi$.

Furthermore, we define

$$
f_{k}(x)=\sum_{j=1}^{k-1} 2^{\frac{k-j}{p}} \psi_{j}(x), \quad x \in \mathbb{R}^{d} .
$$

The proof of (3.23) is finished as soon as we prove that

$$
\left\|f_{k} \mid B_{p q}^{0}\left(\mathbb{R}^{d}\right)\right\| \lesssim k^{\frac{1}{p}} 2^{\frac{k d}{p}}, \quad k \in \mathbb{N},
$$


as well as

$$
\left\|f_{k}\left(2^{k} \cdot\right) \mid B_{p q}^{0}\left(\mathbb{R}^{d}\right)\right\| \gtrsim k^{\frac{1}{q}}, \quad k \in \mathbb{N} .
$$

The proof of (3.26) is a rather straightforward application of Theorem 2.5. We observe, that (3.25) represents an atomic decomposition of $f$. This gives

$$
\left\|f_{k} \mid B_{p q}^{0}\left(\mathbb{R}^{d}\right)\right\| \lesssim\left(\sum_{j=1}^{k-1} 2^{j-1+k(d-1)} 2^{\frac{k-j}{p} \cdot p}\right)^{1 / p} \approx k^{\frac{1}{p}} 2^{\frac{k d}{p}} .
$$

In the proof of (3.27), we use again the characterisation by local means.

We choose a special kernel $\kappa \in C^{\infty}\left(\mathbb{R}^{d}\right)$ with

$$
\operatorname{supp} \kappa \subset[-1,1] \times[-3,3]^{d-1}
$$

and

$$
\kappa(x) \geq 0 \text { if } x_{1} \leq 0 \quad \text { and } \quad \kappa(x)=1 \text { if } x \in\left[-\frac{3}{4}, 0\right] \times[-2,2]^{d-1} .
$$

We show, that for every $j=1, \ldots, k-1$ and every $x \in \mathbb{R}^{d}$ with

$$
2^{-j+1} \leq x_{1} \leq 2^{-j+1}+\frac{2^{-j}}{4}, \quad 0 \leq x_{i} \leq 1, i=2, \ldots, d,
$$

it holds

$$
\mathcal{K}\left(2^{-j}, f\left(2^{k} \cdot\right)\right)(x) \geq c 2^{\frac{j}{p}} .
$$

Let us point out, that this estimate is already sufficient for (3.27) since

$$
\begin{aligned}
\left\|f_{k}\left(2^{k} \cdot\right) \mid B_{p q}^{0}\left(\mathbb{R}^{d}\right)\right\| & \gtrsim\left(\sum_{j=1}^{k-1}\left\|\mathcal{K}\left(2^{-j}, f\left(2^{k} \cdot\right)\right) \mid L_{p}\left(\mathbb{R}^{d}\right)\right\|^{q}\right)^{1 / q} \\
& \gtrsim c\left(\sum_{j=1}^{k-1}\left(2^{-j} 2^{\frac{j}{p} \cdot p}\right)^{p / q}\right)^{1 / q} \approx k^{\frac{1}{q}} .
\end{aligned}
$$

We therefore concentrate on (3.29) under the condition (3.28).

The support properties of $\psi_{j}$ and $\kappa$ ensure, that

$$
\begin{aligned}
\mathcal{K}\left(2^{-j}, f\left(2^{k} \cdot\right)\right)(x) & =2^{\frac{j}{p}} \mathcal{K}\left(2^{-j}, \psi_{k-j}\left(2^{k} \cdot\right)\right)(x) \\
& =2^{\frac{j}{p}} \sum_{l \in N_{k-j}^{k}} \int_{\mathbb{R}^{d}} \kappa(y) \psi\left(2^{k} x+2^{k-j} y-l\right) d y
\end{aligned}
$$


for every $x$ with (3.28). It is not difficult to verify, that (for every $x$ ) there are always at least $2^{(k-j) d}$ vectors $l \in N_{k-j}^{k}$ such that $\kappa(y)=1$ on the support of $\psi\left(2^{k} x+2^{k-j} y-l\right)$. Hence the last expression may be estimated from below by

$$
2^{\frac{j}{p}} \cdot 2^{(k-j) d} \int_{\mathbb{R}^{d}} \psi\left(2^{k-j} y\right) d y=2^{\frac{j}{p}} .
$$

Remark 3.6. Let us observe, that Theorem 3.4 may be easily extended to $0<q<1$ :

$$
\left\|T_{k} \mid \mathcal{L}\left(B_{p q}^{0}\left(\mathbb{R}^{d}\right)\right)\right\| \approx 2^{-k \frac{d}{p}} \cdot \begin{cases}k^{\frac{1}{q}-\frac{1}{p}}, & \text { if } 1<p<\infty \text { and } p \geq \max (q, 2), \\ k^{\frac{1}{q}-\frac{1}{2}}, & \text { if } 1<p<\infty \text { and } 2 \geq \max (p, q), \\ k^{\frac{1}{q}}, & \text { if } p=1 \text { or } p=\infty,\end{cases}
$$

The proof of the estimates from above may be done exactly as in the proof of Theorem 3.2. We use the Gagliardo-Nirenberg inequality, cf. [7, Chapter $5]$,

$$
\left\|f\left|B_{p, 1}^{0}\left(\mathbb{R}^{d}\right)\|\leq\| f\right| B_{p q}^{0}\left(\mathbb{R}^{d}\right)\right\|^{1-\theta} \cdot\left\|f \mid B_{p, \max (p, 2)}^{0}\left(\mathbb{R}^{d}\right)\right\|^{\theta}
$$

with

$$
1=\frac{1-\theta}{q}+\frac{\theta}{\max (p, 2)}
$$

and the construction from the proof of Theorem 3.4 to prove the estimates from below.

Remark 3.7. Theorem 3.4 may also be used to give a following comment on the atomic decomposition Theorem 2.5. If $s=0$, we required in Theorem 2.5 that the atoms $a_{\nu m}$ satisfy the moment condition (2.8) at least for $\beta=0$ and $\nu>0$.

It seems to be an open question, if this restriction is really necessary. In other words, if Theorem 2.5 holds, if $s=0$ as well as $L=-s=0$. We show, that this is never true and that the moment conditions are indispensable.

Let $1<q \leq \infty$ and $1 \leq p \leq \infty$ and let us suppose, that Theorem 2.5 is true with $L=0$. Hence no moment condition on $a_{\nu m}$ are needed. Let $\psi \in S\left(\mathbb{R}^{d}\right)$ be a non-negative function with

$$
\operatorname{supp} \psi \subset\left\{x \in \mathbb{R}^{d}:\left|x_{i}\right| \leq 1, i=1, \ldots, d\right\}, \quad \int_{\mathbb{R}^{d}} \psi(x) d x=1,
$$

and

$$
\sum_{m \in \mathbb{Z}^{d}} \psi(x-m)=1, \quad x \in \mathbb{R}^{d}
$$


We put

$$
f_{J}(x)=\sum_{\nu=0}^{J} \sum_{\substack{m \in \mathbb{Z}^{d} \\\left|m_{i}\right| \leq 2^{\nu}, i=1, \ldots, d}} \psi\left(2^{\nu} x-m\right), \quad x \in \mathbb{R}^{d}, \quad J \in \mathbb{N} .
$$

It follows by (3.32) that

$$
\left\|f_{J} \mid B_{p q}^{0}\left(\mathbb{R}^{d}\right)\right\| \gtrsim J, \quad J \in \mathbb{N} .
$$

But if Theorem 2.5 would be true for $s=0$ and $L=0$, (3.34) would represent an atomic decomposition of $f_{J}$ and therefore

$$
\left\|f_{J} \mid B_{p q}^{0}\left(\mathbb{R}^{d}\right)\right\| \lesssim\left(\sum_{\nu=0}^{J} 2^{-\nu \frac{d}{p} q} \cdot\left(2^{\nu+1}+1\right)^{\frac{d}{p} q}\right)^{1 / q} \lesssim J^{\frac{1}{q}}
$$

would hold for every $J \in \mathbb{N}$. This leads to contradiction.

Let $0<q \leq 1$ and $1 \leq p \leq \infty$. Then every $f \in B_{p q}^{0}\left(\mathbb{R}^{d}\right)$ may be rewritten into the optimal atomic decomposition

$$
f(x)=\sum_{\nu=0}^{\infty} \sum_{m \in \mathbb{Z}^{d}} \lambda_{\nu m} a_{\nu m}(x), \quad x \in \mathbb{R}^{d},
$$

with

$$
\left\|\lambda\left|b_{p q}^{0}\|\lesssim\| f\right| B_{p q}^{0}\left(\mathbb{R}^{d}\right)\right\|, \quad f \in B_{p q}^{0}\left(\mathbb{R}^{d}\right),
$$

see [12, Chapter 1.5] for details. If Theorem 2.5 would be true for $s=0$ and $L=0$,

$$
f\left(2^{k} x\right)=\sum_{\nu=0}^{\infty} \sum_{m \in \mathbb{Z}^{d}} \lambda_{\nu m} a_{\nu m}\left(2^{k} x\right), \quad x \in \mathbb{R}^{d}
$$

would represent an atomic decomposition of $f\left(2^{k} x\right)$ and therefore

$$
\left\|f\left(2^{k} \cdot\right)\left|B_{p q}^{0}\left(\mathbb{R}^{d}\right)\left\|\lesssim 2^{k \frac{d}{p}}\right\| \lambda\right| b_{p q}^{0}\right\| \lesssim 2^{k \frac{d}{p}}\left\|f \mid B_{p q}^{0}\left(\mathbb{R}^{d}\right)\right\| .
$$

But we know by Theorem 3.4 and Remark 3.6 that this is not true.

For the sake of completeness, we consider also the dilation operator

$$
\left(\tilde{T}_{k} f\right)(x)=f\left(2^{-k} x\right), \quad k \in \mathbb{N}, \quad x \in \mathbb{R}^{d} .
$$

Its behaviour is well known if $s<0$, see [4, p. 34] for further details:

Lemma 3.8. Let $s<0,1 \leq p, q \leq \infty$ and $k \in \mathbb{N}$. Then the operator $\tilde{T}_{k}$ is bounded on $B_{p, q}^{s}\left(\mathbb{R}^{d}\right)$ and its norm is bounded by $c 2^{-k\left(s-\frac{d}{p}\right)}$. 
If $s=0$, we can also characterise the norm $\tilde{T}_{k}$.

Theorem 3.9. Let $1 \leq p, q \leq \infty, k \in \mathbb{N}$ and let $\tilde{T}_{k}$ be defined by (3.35). Then

$$
\left\|\tilde{T}_{k} \mid \mathcal{L}\left(B_{p q}^{0}\left(\mathbb{R}^{d}\right)\right)\right\| \approx 2^{k \frac{d}{p}} \cdot \begin{cases}k^{\frac{1}{p}-\frac{1}{q}}, & \text { if } 1<p<\infty \text { and } p \leq \min (q, 2), \\ k^{\frac{1}{2}-\frac{1}{q}}, & \text { if } 1<p<\infty \text { and } 2 \leq \min (p, q), \\ 1, & \text { if } 1<p<\infty \text { and } q \leq \min (p, 2), \\ k^{1-\frac{1}{q},}, & \text { if } p=1 \text { or } p=\infty,\end{cases}
$$

where the constants of equivalence do not depend on $k$.

Remark 3.10. If $1<p<\infty$, the estimates in (3.36) may be abbreviated to

$$
\left\|\tilde{T}_{k} \mid \mathcal{L}\left(B_{p q}^{0}\left(\mathbb{R}^{d}\right)\right)\right\| \approx 2^{k \frac{d}{p}} \cdot k^{\frac{1}{\min (p, q, 2)}-\frac{1}{q}} .
$$

In this case, the jump in the exponent of $k$ occurs by $p=\infty$.

Proof. Let $\stackrel{\circ}{B}_{p q}^{0}\left(\mathbb{R}^{d}\right)$ with $1 \leq p, q \leq \infty$ be the completion of $S\left(\mathbb{R}^{d}\right)$ in $B_{p q}^{0}\left(\mathbb{R}^{d}\right)$. It follows immediately from Theorem 3.4 that

$$
\left\|T_{k}\left|\mathcal{L}\left(B_{p q}^{0}\left(\mathbb{R}^{d}\right)\right)\|=\| T_{k}\right| \mathcal{L}\left(\stackrel{\circ}{B}_{p q}^{0}\left(\mathbb{R}^{d}\right)\right)\right\| .
$$

One has by $[10$, p. $180,(12)]$

$$
\stackrel{\circ}{B}_{p q}^{0}\left(\mathbb{R}^{d}\right)^{\prime}=B_{p^{\prime} q^{\prime}}^{0}\left(\mathbb{R}^{d}\right), \quad 1 \leq p, q \leq \infty \quad \text { and } \quad \frac{1}{p}+\frac{1}{p^{\prime}}=\frac{1}{q}+\frac{1}{q^{\prime}}=1 .
$$

Furthermore

$$
\left\|T_{k}\left|\mathcal{L}\left(\stackrel{\circ}{B}_{p q}^{0}\left(\mathbb{R}^{d}\right)\right)\|=\| T_{k}^{\prime}\right| \mathcal{L}\left(B_{p^{\prime} q^{\prime}}^{0}\left(\mathbb{R}^{d}\right)\right)\right\|
$$

where

$$
T_{k}^{\prime}=2^{-k d} \tilde{T}_{k}
$$

is the dual operator to $T_{k}$. Hence

$$
\left\|\tilde{T}_{k}\left|\mathcal{L}\left(B_{p q}^{0}\left(\mathbb{R}^{d}\right)\right)\left\|=2^{k d}\right\| T_{k}\right| \mathcal{L}\left({\stackrel{\circ}{p^{\prime} q^{\prime}}}^{\prime}\left(\mathbb{R}^{d}\right)\right)\right\| .
$$

Now the proof follows by (3.37) and Theorem 3.4.

It is not difficult to extend Theorems 3.4 and 3.9 also to the operator

$$
\left(T_{\lambda} f\right)(x)=f(\lambda x), \quad \lambda>0, \quad x \in \mathbb{R}^{d} .
$$

Theorem 3.11. Let $1 \leq p, q \leq \infty$. 
(i) Then

$$
\begin{aligned}
& \left\|T_{\lambda} \mid \mathcal{L}\left(B_{p q}^{0}\left(\mathbb{R}^{d}\right)\right)\right\| \\
& \approx \lambda^{-\frac{d}{p}} \cdot \begin{cases}(1+\log \lambda)^{\frac{1}{q}-\frac{1}{p}}, & \text { if } 1<p<\infty \text { and } p \geq \max (q, 2), \\
(1+\log \lambda)^{\frac{1}{q}-\frac{1}{2}}, & \text { if } 1<p<\infty \text { and } 2 \geq \max (p, q), \\
1, & \text { if } 1<p<\infty \text { and } q \geq \max (p, 2), \\
(1+\log \lambda)^{\frac{1}{q}}, & \text { if } p=1 \text { or } p=\infty,\end{cases}
\end{aligned}
$$

holds for every $\lambda>1$.

(ii) Then

$$
\begin{aligned}
& \left\|T_{\lambda} \mid \mathcal{L}\left(B_{p q}^{0}\left(\mathbb{R}^{d}\right)\right)\right\| \\
& \approx \lambda^{-\frac{d}{p}} \cdot \begin{cases}(1+|\log \lambda|)^{\frac{1}{p}-\frac{1}{q}}, & \text { if } 1<p<\infty \text { and } p \leq \min (q, 2), \\
(1+|\log \lambda|)^{\frac{1}{2}-\frac{1}{q}}, & \text { if } 1<p<\infty \text { and } 2 \leq \min (p, q), \\
1, & \text { if } 1<p<\infty \text { and } q \leq \min (p, 2), \\
(1+|\log \lambda|)^{1-\frac{1}{q}}, & \text { if } p=1 \text { or } p=\infty,\end{cases}
\end{aligned}
$$

holds for every $0<\lambda<1$.

Proof. The result follows directly from the Theorems 3.4 and 3.9 and the well-known assertion

$$
\sup _{\frac{1}{2}<\lambda<2}\left\|f(\lambda \cdot)\left|B_{p q}^{0}\left(\mathbb{R}^{d}\right)\|\approx\| f\right| B_{p q}^{0}\left(\mathbb{R}^{d}\right)\right\| .
$$

\section{Sampling numbers}

In this section we apply the estimates of the norm of the dilation operator to derive optimal estimates for the decay of sampling numbers of the identity operator between two Besov spaces. Let us first present the basic definitions and notation.

Definition 4.1. Let $\Omega$ be the unit cube $(0,1)^{d}$. Let $G_{1}(\Omega)$ be a space of continuous functions on $\Omega$ and $G_{2}(\Omega) \subset D^{\prime}(\Omega)$ be a space of distributions on $\Omega$. Suppose, that the embedding

$$
i d: G_{1}(\Omega) \hookrightarrow G_{2}(\Omega)
$$

is compact. 
For $\left\{x^{j}\right\}_{j=1}^{n} \subset \Omega$ we define the information map

$$
N_{n}: G_{1}(\Omega) \rightarrow \mathbb{C}^{n}, \quad N_{n} f=\left(f\left(x^{1}\right), \ldots, f\left(x^{n}\right)\right), \quad f \in G_{1}(\Omega) .
$$

For any (linear or nonlinear) mapping $\varphi_{n}: \mathbb{C}^{n} \rightarrow G_{2}(\Omega)$ we consider

$$
S_{n}: G_{1}(\Omega) \rightarrow G_{2}(\Omega), \quad S_{n}=\varphi_{n} \circ N_{n} .
$$

(i) Then for all $n \in \mathbb{N}$, the $n$-th sampling number $g_{n}(i d)$ is defined by

$$
g_{n}(i d)=\inf _{S_{n}} \sup \left\{\left\|f-S_{n} f\left|G_{2}(\Omega)\|:\| f\right| G_{1}(\Omega)\right\| \leq 1\right\},
$$

where the infimum is taken over all $n$-tuples $\left\{x^{j}\right\}_{j=1}^{n} \subset \Omega$ and all (linear or nonlinear) $\varphi_{n}$.

(ii) For all $n \in \mathbb{N}$ the $n$-th linear sampling number $g_{n}^{\text {lin }}(i d)$ is defined by (4.1), where now only linear mappings $\varphi_{n}$ are admitted.

In the following, we restrict ourselves to the scale of Besov spaces - hence $G_{1}(\Omega)=B_{p_{1} q_{1}}^{s_{1}}(\Omega)$ with

$$
s_{1}>\frac{d}{p_{1}} \text {. }
$$

Then the space $B_{p_{1} q_{1}}^{s}(\Omega)$ is continuously embedded into the space of functions continuous on $\bar{\Omega}$ and the information map $N_{n}$ is well defined. Second, we suppose that $G_{2}=B_{p_{2} q_{2}}^{0}(\Omega)$.

The case $p_{1}<p_{2}$ was already fully discussed in [13]. It was shown there, that both, the linear and nonlinear sampling numbers, decay asymptotically like $n^{-\frac{s}{d}+\frac{1}{p_{1}}-\frac{1}{p_{2}}}$.

We concentrate on the case $p_{1}=p_{2}$ and give a full characterisation of the decay of $g_{n}$ as well as of $g_{n}^{\text {lin }}$. This result closes some of the gaps left open in [13], which were the actual motivation for this paper. In the very end, we discuss the remaining case $p_{1}>p_{2}$ and state several open problems connected to this question.

Theorem 4.2. Let $\Omega=(0,1)^{d}$. Let $G_{1}(\Omega)=B_{p q_{1}}^{s}(\Omega)$ and $G_{2}(\Omega)=$ $B_{p q_{2}}^{0}(\Omega)$ with $1 \leq p, q_{1}, q_{2} \leq \infty$ and $s>\frac{d}{p}$. Then for $2 \leq n \in \mathbb{N}$

$$
\begin{aligned}
g_{n}(i d) \approx g_{n}^{\operatorname{lin}}(i d) \\
\approx n^{-\frac{s}{d}} \cdot \begin{cases}(\log n)^{\frac{1}{q_{2}}-\frac{1}{p}}, & \text { if } 1<p<\infty \text { and } p \geq \max \left(q_{2}, 2\right), \\
(\log n)^{\frac{1}{q_{2}}-\frac{1}{2}}, & \text { if } 1<p<\infty \text { and } 2 \geq \max \left(p, q_{2}\right), \\
1, & \text { if } 1<p<\infty \text { and } q_{2} \geq \max (p, 2), \\
(\log n)^{\frac{1}{q_{2}}}, & \text { if } p=1 \text { or } p=\infty,\end{cases}
\end{aligned}
$$

Proof. Step 1: Estimates from above 
It follows directly from Definition 4.1 that $g_{n}(i d) \leq g_{n}^{\operatorname{lin}}(i d)$. The estimates from above for $g_{n}^{\text {lin }}$ are a consequence of the estimates from above obtained in Lemma 3.1 and Proposition 3.2 and summarised in Theorem 3.4 and the method presented in [13]. By this we mean especially the inequality (2.6) in [13] where now the estimate of the norm of the dilation operator has to be applied with $s_{2}=0$.

Hence, it is enough to prove the estimates from below for $g_{n}(i d)$.

Step 2. - Estimates from below

We use the following simple observation, (c.f. [6, Proposition 20]). For $\Gamma=\left\{x^{j}\right\}_{j=1}^{n} \subset \Omega$ we denote

$$
G_{1}^{\Gamma}(\Omega)=\left\{f \in G_{1}(\Omega): f\left(x^{j}\right)=0 \quad \text { for all } \quad j=1, \ldots, n\right\} .
$$

Then

$$
\begin{aligned}
g_{n}(i d) & \approx \inf _{\Gamma} \sup \left\{\left\|f\left|G_{2}(\Omega)\left\|: f \in G_{1}^{\Gamma}(\Omega),\right\| f\right| G_{1}(\Omega)\right\|=1\right\} \\
& =\inf _{\Gamma}\left\|i d: G_{1}^{\Gamma}(\Omega) \hookrightarrow G_{2}(\Omega)\right\|,
\end{aligned}
$$

where both the infima are taken over all sets $\Gamma=\left\{x^{j}\right\}_{j=1}^{n} \subset \Omega$.

So, to prove the estimates from below included in (4.2), we construct for every set $\Gamma=\left\{x^{j}\right\}_{j=1}^{2^{k d}} \subset \Omega, k \in \mathbb{N}$ a function $f_{k} \in G_{1}^{\Gamma}$ such that

$$
\frac{\left\|f_{k} \mid G_{2}(\Omega)\right\|}{\left\|f_{k} \mid G_{1}(\Omega)\right\|} \gtrsim 2^{-k s} k^{\alpha}
$$

where the power $\alpha$ represents the power of the logarithmic factor in each of the four cases contained in (4.2).

1. case: $g_{n}(i d) \gtrsim n^{-\frac{s}{d}}$.

In this (most simple) case, we rely on the wavelet characterisation of the spaces $B_{p q}^{s}\left(\mathbb{R}^{d}\right)$, as described in [12, Section 3.1]. Let

$$
\psi_{F} \in C^{K}(\mathbb{R}) \quad \text { and } \quad \psi_{M} \in C^{K}(\mathbb{R}), \quad K \in \mathbb{N},
$$

be the Daubechies compactly supported $K$-wavelets on $\mathbb{R}$ with $K$ large enough. Then we define

$$
\Psi(x)=\prod_{i=1}^{d} \psi_{M}\left(x_{i}\right), \quad x=\left(x_{1}, \ldots, x_{d}\right) \in \mathbb{R}^{d}
$$

and

$$
\Psi_{m}^{j}(x)=\Psi\left(2^{j} x-m\right), \quad j \in \mathbb{N}_{0}, \quad m \in \mathbb{Z}^{d} .
$$


The functions

$$
\psi_{j}(x)=\sum_{m} \lambda_{j m} \Psi_{m}^{j}(x), \quad j \in \mathbb{N}, \quad x \in \mathbb{R}^{d}
$$

satisfy

$$
\left\|\psi_{j} \mid B_{p q}^{s}\left(\mathbb{R}^{d}\right)\right\| \approx 2^{j\left(s-\frac{d}{p}\right)}\left(\sum_{m}\left|\lambda_{j m}\right|^{p}\right)^{1 / p}
$$

with constants independent on $j \in \mathbb{N}$ and on the sequence $\lambda=\left\{\lambda_{j m}\right\}$. The summation in (4.4) and (4.5) runs over those $m \in \mathbb{Z}^{d}$ for which the support of $\Psi_{m}^{j}$ is included in $\Omega$. Let us comment briefly on the relationship between $\left\|\psi_{j} \mid B_{p q}^{s}(\Omega)\right\|$ and $\left\|\psi_{j} \mid B_{p q}^{s}\left(\mathbb{R}^{d}\right)\right\|$. Clearly, $\psi_{j}$ as a function on $\mathbb{R}^{d}$ is an extension of $\left.\psi_{j}\right|_{\Omega}$, the inequality

$$
\left\|\psi_{j}\left|B_{p q}^{s}(\Omega)\|\leq\| \psi_{j}\right| B_{p q}^{s}\left(\mathbb{R}^{d}\right)\right\|
$$

follows trivially from Definition 2.7. On the other hand, any other extension of $\left.\psi_{j}\right|_{\Omega}$ to $\mathbb{R}^{d}$ possesses an unique wavelet decomposition. The uniqueness shows, that this decomposition contains (4.4) as a proper part and has therefore a larger norm. Hence, the relation (4.5) holds also for $\left\|\psi_{j} \mid B_{p q}^{s}(\Omega)\right\|$.

There is a number $l \in \mathbb{N}_{0}$ such that for any $k \in \mathbb{N}$ and any $\Gamma=\left\{x^{j}\right\}_{j=1}^{2^{k d}}$ there is an element $m \in \mathbb{Z}^{d}$ such that

$$
\operatorname{supp} \Psi_{m}^{k+l} \subset \Omega \text { and } \operatorname{supp} \Psi_{m}^{k+l} \cap \Gamma=\emptyset .
$$

Taking $f_{k}=\Psi_{m}^{k+l}$ we obtain the estimate $g_{n}(i d) \gtrsim n^{-\frac{s}{d}}$ for every $1 \leq p, q_{1}, q_{2} \leq \infty$.

2. case: $g_{n}(i d) \gtrsim n^{-\frac{s}{d}}(\log n)^{\frac{1}{q_{2}}}$ for $p=1$.

We consider the function $\psi_{k}(x)=\psi\left(2^{k} x-m\right)$, where $m \in \mathbb{Z}^{d}$ and $\psi$ was defined and discussed in the Step 1. of the proof of Theorem 3.4. It is possible to choose $m \in \mathbb{Z}^{d}$ such that

$$
\operatorname{supp} \psi_{k} \subset\left(\frac{1}{4}, \frac{3}{4}\right)^{d} \quad \text { and } \quad \operatorname{supp} \psi_{k} \cap \Gamma=\emptyset .
$$

To show, that this function satisfies (4.3), we argue as follows. First, we use Theorem 2.5 to get

$$
\left\|\psi_{k}\left|B_{1 q_{1}}^{s}(\Omega)\|\leq\| \psi_{k}\right| B_{1 q_{1}}^{s}\left(\mathbb{R}^{d}\right)\right\| \lesssim 2^{k(s-d)} .
$$


On the other hand, if $\tilde{\psi}_{k}$ is any extension of $\psi_{k}$ and $\omega \in S\left(\mathbb{R}^{d}\right)$ satisfies

$$
\operatorname{supp} \omega \subset(0,1)^{d} \text { and } \omega(x)=1 \quad \text { for } \quad x \in\left(\frac{1}{4}, \frac{3}{4}\right)^{d} \text {, }
$$

we arrive at

$$
k^{\frac{1}{q_{2}}} 2^{-k d} \lesssim\left\|\psi_{k}\left|B_{1 q_{2}}^{0}\left(\mathbb{R}^{d}\right)\|=\| \omega \tilde{\psi}_{k}\right| B_{1 q_{2}}^{0}\left(\mathbb{R}^{d}\right)\right\| \lesssim\left\|\tilde{\psi}_{k} \mid B_{1 q_{2}}^{0}\left(\mathbb{R}^{d}\right)\right\|,
$$

hence

$$
k^{\frac{1}{q_{2}}} 2^{-k d} \lesssim\left\|\psi_{k} \mid B_{1 q_{2}}^{0}(\Omega)\right\|
$$

and (4.3) follows.

3. case: $g_{n}(i d) \gtrsim n^{-\frac{s}{d}}(\log n)^{\frac{1}{q_{2}}-\frac{1}{p}}$.

Let $1 \leq p \leq \infty$. In Step 5 . of the proof of Theorem 3.4 we constructed a function $f_{k}\left(2^{k}\right.$.) (see (3.25) for details). Let us point out, that this function has its support in $(0,1)^{d}$ and avoids the set $\Gamma$ if the sampling points are uniformly distributed, hence $\Gamma=\left\{0, \frac{1}{2^{k}}, \ldots, \frac{2^{k}-1}{2^{k}}, 1\right\}^{d}$. Using (3.26) and (3.27), we obtain

$$
\left\|f\left(2^{k} \cdot\right)\left|B_{p q_{1}}^{s}\left(\mathbb{R}^{d}\right)\left\|\lesssim 2^{k\left(s-\frac{d}{p}\right)}\right\| f\right| B_{p q_{1}}^{s}\left(\mathbb{R}^{d}\right)\right\| \lesssim 2^{k\left(s-\frac{d}{p}\right)} 2^{\frac{k d}{p}} k^{\frac{1}{p}}=k^{\frac{1}{p}} 2^{k s}
$$

and

$$
\left\|f\left(2^{k} \cdot\right) \mid B_{p q_{2}}^{0}\left(\mathbb{R}^{d}\right)\right\| \gtrsim k^{\frac{1}{q_{2}}} .
$$

Using again the cut-off function $\omega$, we get similar estimates also for the norms on $\Omega$. In view of (4.3), this finishes the proof for this specially chosen set $\Gamma$.

If $\Gamma$ is taken arbitrary, $|\Gamma|=2^{k d}$, we modify $f_{k}$ using the Dirichlet principle. Let us sketch this modification.

First, we construct a sequence of disjoint cubes

$$
\left\{\Omega_{j, l}\right\}, \quad j=1, \ldots, k, \quad l=1, \ldots, 2^{(d-1)(j-1)},
$$

where each $\Omega_{j, l}$ is a cube with side length $1 / 2^{j+1}$ and contains in its interior at most $2^{(k-j) d}$ points from $\Gamma$.

We proceed by induction. Let $j=1$. We divide $\Omega=(0,1)^{d}$ into $4^{d}$ cubes with side length $1 / 4$ and disjoint interiors. According to the Dirichlet principle, one of this cubes has in its interior at most $\frac{2^{k d}}{4^{d}}=2^{(k-2) d} \leq$ $2^{(k-1) d}$ points from $\Gamma$. We denote this cube $\Omega_{1,1}$.

Let $j=2$. We divide each of the remaining $4^{d}-1$ cubes (it means the set $\left.\Omega \backslash \Omega_{1,1}\right)$ into $2^{d}$ cubes with side length $1 / 8$ and disjoint interiors. We choose from these $2^{3 d}-2^{d}$ cubes $2^{d-1}$ cubes with the smallest number of 
points of $\Gamma$. The Dirichlet principle gives the estimate from above for this number by $\frac{2^{k d}}{2^{3 d}-2^{d}-2^{d-1}+1} \leq 2^{(k-2) d}$.

In next steps we always divide all remaining cubes into $2^{d}$ cubes with disjoint interiors and half the side length and choose those $2^{(j-1)(d-1)}$ of them which contain the smallest number of points of $\Gamma$. The Dirichlet principle then provides the estimate for this number.

Next, we divide each of the cubes $\Omega_{j, l}$ into $3^{d}$ cubes with disjoint interior and denote 'the middle cube' of this decomposition by $\tilde{\Omega}_{j, l}$.

As each of the cubes $\tilde{\Omega}_{j, l}$ contains at most $2^{(k-j) d}$, there is a number $m>0$ such that we may place into each $\Omega_{j, l} 2^{j d}$ copies (i.e. dilations) of $\psi\left(2^{m+k}\right.$. $)$ with disjoint supports. We denote their sum as $\psi_{j, l}$. The number $m$ may be chosen independent of $k$ and $\Gamma$.

Finally, we introduce

$$
g_{k}(x)=\sum_{j=1}^{k} \sum_{l=1}^{2^{(d-1)(j-1)}} 2^{\frac{j}{p}} \psi_{j l}(x) .
$$

The functions $g_{k}$ play the role of a substitute of $f_{k}\left(2^{k} \cdot\right)$ adapted to the general sampling sets $\Gamma$.

To finish the proof, we have to show that

$$
\left\|g_{k} \mid B_{p q_{2}}^{0}\left(\mathbb{R}^{d}\right)\right\| \gtrsim k^{\frac{1}{q_{2}}}
$$

and

$$
\left\|g_{k} \mid B_{p q_{1}}^{s}\left(\mathbb{R}^{d}\right)\right\| \lesssim k^{\frac{1}{p}} 2^{k s}
$$

The proof of (4.7) is similar to Step 5. of Theorem 3.4 and uses the characterisation by local mean. The proof of (4.8) is based on the atomic decomposition of the spaces $B_{p q_{1}}^{s}\left(\mathbb{R}^{d}\right)$. Let us mention, that $s>0$ and hence no moment conditions are needed in (2.8).

4. case $g_{n}(i d) \gtrsim n^{-\frac{s}{d}}(\log n)^{\frac{1}{q_{2}}-\frac{1}{2}}$.

We first present a construction which proves the result for $d=1$, $\Omega=(-2,2)$ and the uniform distribution of sampling points, i. e. $\Gamma=\left\{\frac{n}{2^{k}}, n=-2^{k+1}+1, \ldots 2^{k+1}-1\right\}$.

We proceed as follows. First, we define a sequence of sets. Let (see Figure 1)

$$
\begin{aligned}
& I_{1}=\left(-\frac{1}{2}, \frac{1}{2}\right), \\
& I_{2}=\left(-\frac{5}{4},-\frac{3}{4}\right) \cup\left(-\frac{1}{4}, \frac{1}{4}\right) \cup\left(\frac{3}{4}, \frac{5}{4}\right),
\end{aligned}
$$




$$
\begin{aligned}
I_{3}= & \left(-\frac{13}{8},-\frac{11}{8}\right) \cup\left(-\frac{9}{8},-\frac{7}{8}\right) \cup\left(-\frac{5}{8},-\frac{3}{8}\right) \cup\left(-\frac{1}{8}, \frac{1}{8}\right) \cup \\
& \cup\left(\frac{3}{8}, \frac{5}{8}\right) \cup\left(\frac{7}{8}, \frac{9}{8}\right) \cup\left(+\frac{11}{8},+\frac{13}{8}\right), \\
\vdots & \bigcup\left\{\left(\frac{4 k-1}{2^{n}}, \frac{4 k+1}{2^{n}}\right) ;|k|<2^{n}\right\},
\end{aligned}
$$

and

$$
I_{n}^{c}=\left(-2+\frac{3}{2^{n}}, 2-\frac{3}{2^{n}}\right) \backslash I_{n} .
$$

Let

$$
\eta_{i}=\chi_{I_{i}}-\chi_{I_{i}^{c}} .
$$

Observe that

$$
\left\langle\eta_{i} ; \eta_{j}\right\rangle= \begin{cases}0, & i \neq j, \\ 2-\frac{1}{2^{i-1}}, & i=j .\end{cases}
$$

The functions $\eta_{i}$ are modified Rademacher functions. Slight modification of Theorem 2.b.3 in Volume I of [5] shows that Khintchin inequalities apply to these functions. Especially, for every $p<\infty$ there is a constant $B_{p}$ such that

$$
\left\|\sum_{i=1}^{k} \eta_{i} \mid L_{p}(\mathbb{R})\right\| \leq B_{p} k^{\frac{1}{2}}
$$

for every $k \in \mathbb{N}$.

Now, take a non-negative non-trivial function $\kappa \in S(\mathbb{R})$ with $\operatorname{supp} \kappa \subset$ $(0,1)$. As $I_{i}$ contains $2^{i}-1$ intervals of the length $\frac{2}{2^{i}}$, we may define the functions $g_{k, i}, i=1, \ldots, k$, as the sum of $2^{k}\left(2-2^{-(i-1)}\right)$ copies of the function $\kappa\left(2^{k}\right.$.) with disjoint supports all contained in $I_{i}$. Similarly, $g_{k, i}^{c}, i=1, \ldots, k$, is the sum of $2^{k}\left(2-2^{-(i-2)}\right)$ copies of $\kappa\left(2^{k} \cdot\right)$ with disjoint supports all contained in $I_{i}^{c}$. We define

$$
g_{k}=\sum_{i=1}^{k}\left(g_{k, i}-g_{k, i}^{c}\right) \text {. }
$$

The atomic decomposition theorem (cf. Theorem 2.5) together with (4.9) yields

$$
\left\|g_{k}\left|B_{p, q_{1}}^{s}(\mathbb{R})\|\lesssim\| 2^{k s} \sum_{i=1}^{k} \eta_{i}\right| L_{p}(\mathbb{R})\right\| \lesssim k^{\frac{1}{2}} 2^{k s}, \quad k \in \mathbb{N} .
$$


To estimate the norm of $g_{k}$ in $B_{p, q_{2}}^{0}(\mathbb{R})$ from below, we use duality.

Set

$$
\tilde{\kappa}_{i}(x)=\kappa\left(2^{i} x\right)-\kappa\left(2^{i} x-1\right), \quad x \in \mathbb{R}, \quad i \in \mathbb{N} .
$$

We define the functions $\tilde{g}_{i}$ as the sum of $2^{i}-2$ copies of $\tilde{\kappa}_{i}$ with disjoint supports all contained in $I_{i} \cup I_{i}^{c}$, non-negative on $I_{i}$, non-positive on $I_{i}^{c}$. Finally, we write

$$
\tilde{g}^{k}=\sum_{i=2}^{k} \tilde{g}_{i}, \quad k \geq 2 .
$$

An application of the atomic decomposition theorem 2.5 leads to

$$
\left\|\tilde{g}^{k} \mid B_{p^{\prime}, q_{2}^{\prime}}^{0}(\mathbb{R})\right\| \lesssim k^{\frac{1}{q_{2}^{\prime}}}=k^{1-\frac{1}{q_{2}}} .
$$

Let us mention, that the first moment condition $\int_{\mathbb{R}} \kappa(x) d x=0$ is satisfied trivially by (4.10). Now we apply the functional represented by $g_{k}$ to $\tilde{g}^{k}$. Then

$$
\begin{aligned}
k & \approx \int_{-2}^{2} g_{k}(t) \tilde{g}^{k}(t) d t \\
& =g_{k}\left(\tilde{g}^{k}\right) \\
& \lesssim\left\|g_{k}\left|B_{p, q_{2}}^{0}(\mathbb{R})\|\cdot\| \tilde{g}^{k}\right| B_{p^{\prime}, q_{2}^{\prime}}^{0}(\mathbb{R})\right\| \\
& \lesssim k^{1-\frac{1}{q_{2}}}\left\|g_{k} \mid B_{p, q_{2}}^{0}(\mathbb{R})\right\|,
\end{aligned}
$$

which implies

$$
k^{\frac{1}{q_{2}}} \lesssim\left\|g_{k} \mid B_{p, q_{2}}^{0}(\mathbb{R})\right\|, \quad k \in \mathbb{N} .
$$

Let us point out, that the function $g_{k}$ vanishes on $\Gamma$. In view of (4.3), this finishes the proof for $d=1$ and uniform distribution of the sampling points.

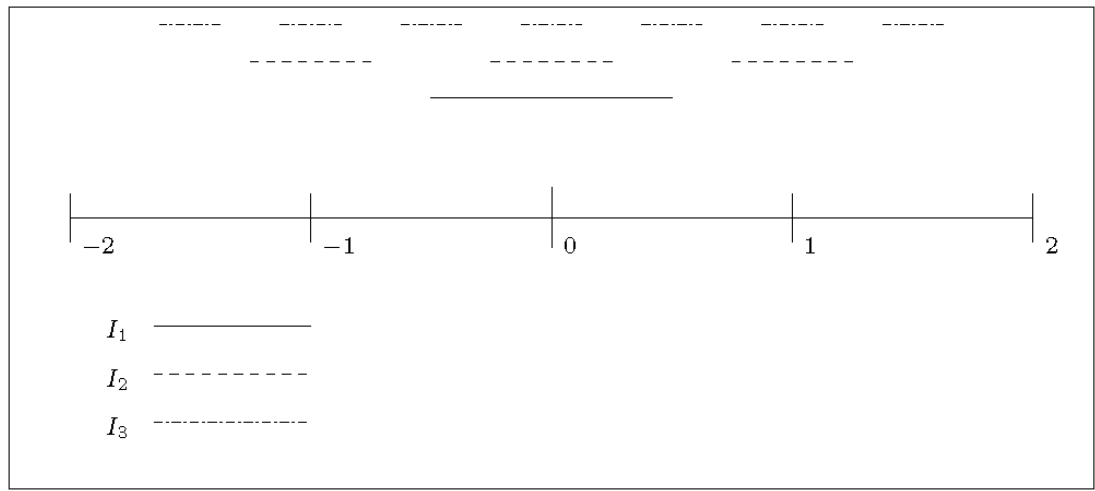

Figure 1 
If the sampling points are not uniformly distributed, the construction has to be slightly modified. Let $\Omega=(0,1), k \in \mathbb{N}$ and let $\Gamma \subset(0,1)$ be an arbitrary set with $\# \Gamma \leq 2^{k}$. We denote by $I_{j}^{k}$ the dyadic decomposition of $(0,1)$ into $2^{k}$ disjoint intervals of length $2^{-k}$, hence

$$
I_{j}^{k}=\left(\frac{j}{2^{k}}, \frac{j+1}{2^{k}}\right), \quad j=0, \ldots, 2^{k}-1 .
$$

Furthermore, $\tilde{\Gamma}_{k}$ stands for the union of intervals $I_{j}^{k}$, which intersect $\Gamma$

$$
\tilde{\Gamma}_{k}=\left\{\bigcup_{j} I_{j}^{k}: I_{j}^{k} \cap \Gamma \neq \emptyset\right\} .
$$

Let $r_{j}, j=1,2, \ldots$ be the usual Rademacher functions

$r_{1}(t)=\left\{\begin{array}{ll}1, & \text { if } 0<t<\frac{1}{2}, \\ -1, & \text { if } \frac{1}{2}<t<1, \\ 0 & \text { otherwise }\end{array}\right.$ and $r_{j+1}(t)=r_{j}(2 t)+r_{j}(2 t-1), j=1,2, \ldots$

We set

$$
R_{k}(t)=\sum_{j=1}^{k} r_{j}(t), \quad k \in \mathbb{N}
$$

and

$$
g_{k, i}(t)=r_{i}(t) \cdot \sum_{j=0}^{2^{k}-1} \kappa\left(2^{k} t-j\right), \quad i=1,2, \ldots, k,
$$

where $\kappa \in C^{\infty}(\mathbb{R})$ is a non-trivial non-negative function with $\operatorname{supp} \kappa \subset$ $(0,1)$. Finally, for $a \in \mathbb{N}$ we define

$$
g_{k}^{a}(t)=\left(\sum_{i=1}^{k} g_{k+a, i}(t)\right) \cdot\left(1-\chi_{\tilde{\Gamma}_{k+a}}(t)\right) .
$$

We prove that, if $a$ is chosen sufficiently large and $1<p \leq 2$,

$$
\left\|g_{k}^{a} \mid B_{p, q_{1}}^{s}(\Omega)\right\| \lesssim k^{\frac{1}{2}} 2^{k s}, \quad k \in \mathbb{N}
$$

and

$$
\left\|g_{k}^{a} \mid B_{p, q_{2}}^{0}(\Omega)\right\| \gtrsim k^{\frac{1}{q_{2}}}, \quad k \in \mathbb{N} .
$$

To prove (4.12), we use Theorem 2.5

$$
\left\|g_{k}^{a}\left|B_{p, q_{1}}^{s}(\Omega)\left\|^{2} \leq\right\| g_{k}^{a}\right| B_{p, q_{1}}^{s}(\mathbb{R})\right\|^{2} \lesssim 2^{2(k+a) s}|| R_{k}(t) \cdot\left(1-\chi_{\tilde{\Gamma}_{k+a}}\right) \mid L_{p}(\mathbb{R}) \|^{2}
$$




$$
\begin{aligned}
& \lesssim 2^{2 k s}\left\|R_{k}(t) \cdot\left(1-\chi_{\tilde{\Gamma}_{k+a}}\right) \mid L_{2}(\mathbb{R})\right\|^{2} \\
& =2^{2 k s} \sum_{i, j=1}^{k}\left(r_{i}, r_{j}\right)-2^{2 k s} \sum_{i, j=1}^{k}\left(r_{i}, r_{j} \chi_{\tilde{\Gamma}_{k+a}}\right) .
\end{aligned}
$$

The first sum is obviously equal to $k \cdot 2^{2 k s}$. We rewrite the second sum

$$
\sum_{i, j=1}^{k}\left(r_{i}, r_{j} \chi_{\tilde{\Gamma}_{k+a}}\right)=\sum_{l: I_{l}^{k+a} \subset \tilde{\Gamma}_{k+a}} \int_{I_{l}^{k+a}} \sum_{i, j=1}^{k} r_{i}(t) r_{j}(t) d t
$$

We fix an interval $I_{l}^{k+a} \subset \tilde{\Gamma}_{k+a}$ an observe that the Rademacher functions $r_{i}, i=1, \ldots, k$, are identically +1 or -1 on $I_{l}^{k+a}$. We denote by $\beta_{l}^{+}$the number of those functions, which are identically +1 on $I_{l}^{k+a}$, and similarly for $\beta_{l}^{-}=k-\beta_{l}^{+}$. Then

$\sum_{i, j=1}^{k} r_{i}(t) r_{j}(t)=\beta_{l}^{+} \cdot \beta_{l}^{+}+\beta_{l}^{-} \cdot \beta_{l}^{-}-2 \beta_{l}^{+} \cdot \beta_{l}^{-}=\left(\beta_{l}^{+}-\beta_{l}^{-}\right)^{2} \geq 0, \quad t \in I_{l}^{k+a}$.

Hence, the last sum in (4.14) is always non-negative. This finishes the proof of (4.12).

To prove (4.13), we use duality. We prove that (for $1<p \leq 2$ and $\left.1<q_{2} \leq 2\right)$

$$
\left\|R_{k} \mid B_{p^{\prime} q_{2}^{\prime}}^{0}(\Omega)\right\| \lesssim k^{\frac{1}{q_{2}^{\prime}}}, \quad k \in \mathbb{N}
$$

and

$$
k \lesssim \int_{0}^{1} g_{k}^{a}(t) R_{k}(t) d t, \quad k \in \mathbb{N} .
$$

From (4.15) and (4.16), the result follows similarly to (4.11). For $1<p \leq 2$ and $q_{2}=1$, we use the Gagliardo-Nirenberg inequality

$$
k^{\frac{1}{q}} \lesssim\left\|g_{k}^{a}\left|B_{\tilde{p}, \tilde{q}}^{0}(\Omega)\|\lesssim\| g_{k}^{a}\right| B_{p, 1}^{0}(\Omega)\right\|^{1-\theta} \cdot\left\|g_{k}^{a} \mid B_{2,2}^{0}(\Omega)\right\|^{\theta}
$$

with

$$
0<\theta<1, \quad \frac{1}{\tilde{p}}=\frac{1-\theta}{p}+\frac{\theta}{2}, \quad \frac{1}{\tilde{q}}=\frac{1-\theta}{1}+\frac{\theta}{2}
$$

and the estimate $\left\|g_{k}^{a}\left|B_{2,2}^{0}(\Omega)\|\approx\| g_{k}^{a}\right| L_{2}(\Omega)\right\| \lesssim k^{\frac{1}{2}}$. 
Let us comment on (4.15) and (4.16). The proof of (4.15) may be based on local means, or the reader may consult [9]. To prove (4.16) we write

$$
\begin{aligned}
\int_{0}^{1} g_{k}^{a}(t) R_{k}(t) d t & =\int_{0}^{1}\left(\sum_{i=1}^{k} g_{k+a, i}(t)\right) \cdot\left(1-\chi_{\tilde{\Gamma}_{k+a}}(t)\right) \cdot\left(\sum_{j=1}^{k} r_{j}(t)\right) d t \\
& =\sum_{i, j=1}^{k} \int_{0}^{1} g_{k+a, i}(t) r_{j}(t) d t-\sum_{i, j=1}^{k} \int_{\tilde{\Gamma}_{k+a}} g_{k+a, i}(t) r_{j}(t) d t \\
& =k\left\|\kappa\left|L_{1}(\mathbb{R})\|-\| \kappa\right| L_{1}(\mathbb{R})\right\| \sum_{i, j=1}^{k} \int_{\tilde{\Gamma}_{k+a}} r_{i}(t) r_{j}(t) d t .
\end{aligned}
$$

Using (4.14) one may show that

$$
\sum_{i, j=1}^{k} \int_{\tilde{\Gamma}_{k+a}} r_{i}(t) r_{j}(t) d t \leq c k, \quad k \in \mathbb{N}
$$

with $c<1$. This calculation gives also the only restriction on $a$ and it turns out, that $a=2$ will do the job. This finishes the proof in $d=1$.

If $d>1$, only minor modifications using tensor products are needed. We leave out the details.

Remark 4.3. This result describes the decay of (linear and nonlinear) sampling numbers of the embedding

$$
i d: B_{p_{1} q_{1}}^{s}(\Omega) \rightarrow B_{p_{2} q_{2}}^{0}(\Omega)
$$

if $p_{1}=p_{2}$. The results for $p_{1}<p_{2}$ may be easily derived from [13], the sampling numbers decay like $n^{-\frac{s_{1}}{d}+\left(\frac{1}{p_{1}}-\frac{1}{p_{2}}\right)}$. If $p_{1}>p_{2}$, we may use one of the embeddings

$$
\begin{aligned}
& B_{p_{1} q_{1}}^{s}(\Omega) \hookrightarrow B_{p_{1} q_{2}}^{0}(\Omega) \hookrightarrow B_{p_{2} q_{2}}^{0}(\Omega), \\
& B_{p_{1} q_{1}}^{s}(\Omega) \hookrightarrow B_{p_{2} q_{1}}^{s}(\Omega) \hookrightarrow B_{p_{2} q_{2}}^{0}(\Omega)
\end{aligned}
$$

and obtain (some) estimates from above. Using some of the "test functions" mentioned above, we may also provide certain estimates from below. But it should be pointed out, that in several cases, there is a logarithmic gap between the estimates from above and the estimates from below. We leave the detailed discussion opened and do not state the partial results. 


\section{References}

[1] G. Bourdaud, Sur les opérateurs pseudo-différentiels à coefficinets peu réguliers, Habilitation thesis, Université de Paris-Sud, Paris, 1983.

[2] H.-Q. Bui, M. Paluszyński and M. H. Taibleson, A maximal function characterization of weighted Besov-Lipschitz and Triebel-Lizorkin spaces, Studia Math., 119 (1996), 219-246.

[3] H.-Q. Bui, M. Paluszyński and M. H. Taibleson, Characterization of weighted Besov-Lipschitz and Triebel-Lizorkin spaces. The case $q<1$, Journ. Fourier Anal. Appl., 3 (Special issue) (1997), 837-846.

[4] D. E. Edmunds and H. Triebel, Function spaces, entropy numbers, differential operators, Cambridge Univ. Press, Cambridge, 1996.

[5] J. Lindenstrauss and L. Tzafriri, Classical Banach Spaces I and II, Springer, 1996.

[6] E. Novak and H. Triebel, Function spaces in lipschitz domains and optimal rates of convergence for sampling, Constr. Approx., 23 (2006), 325-350.

[7] J. Peetre, New Thoughts on Besov Spaces, Duke Univ. Math. Series, Durham, Univ., 1976.

[8] V. S. Rychkov, On a theorem of Bui, Paluszyński and Taibleson, Proc. Steklov Inst. Math., 227 (1999), 280-292.

[9] H. Triebel, On Haar bases in Besov spaces, Serdica 4 (1978), 330-343.

[10] H. Triebel, Theory of Function Spaces, Birkhäuser, Basel, 1983.

[11] H. Triebel, Theory of Function Spaces II, Birkhäuser, Basel, 1992.

[12] H. Triebel, Theory of Function Spaces III, Birkhäuser, Basel, 2006.

[13] J. Vybíral, Sampling numbers and function spaces, J. Compl., 23 (2007), 773-792.

Mathematisches Institut

Friedrich-Schiller-Universität Jena

Ernst-Abbe-Platz 3

07740 Jena

Germany

(E-mail : vybiral@minet.uni-jena.de)

(Received: May 2007) 


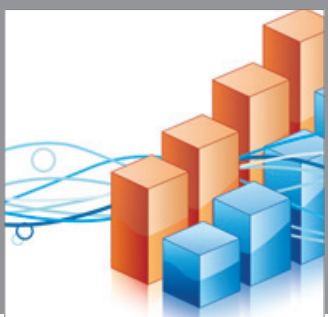

Advances in

Operations Research





\section{The Scientific} World Journal
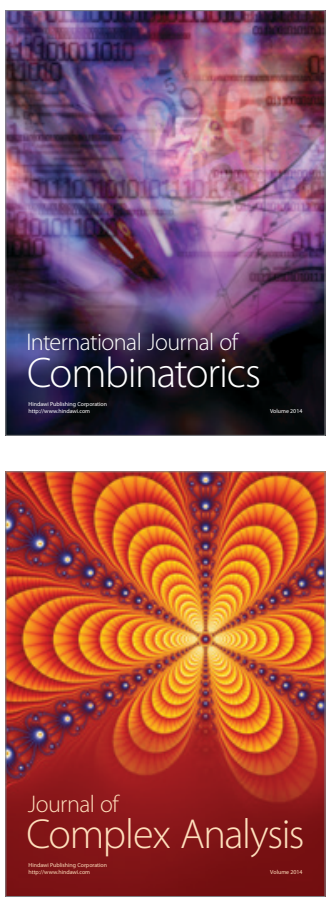

International Journal of

Mathematics and

Mathematical

Sciences
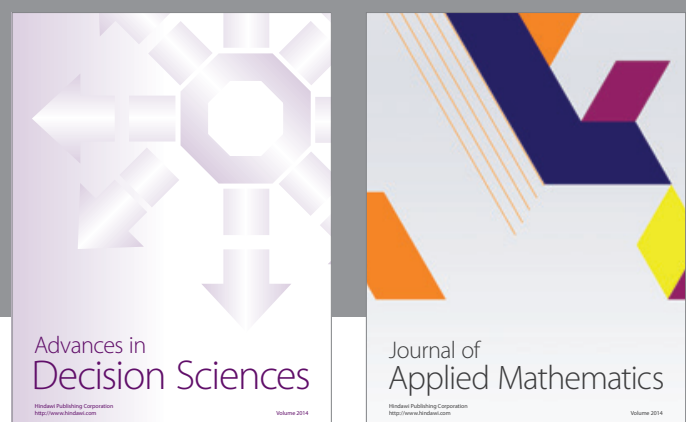

Journal of

Applied Mathematics
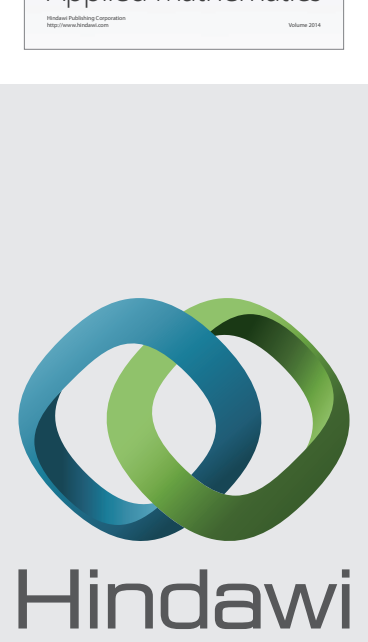

Submit your manuscripts at http://www.hindawi.com
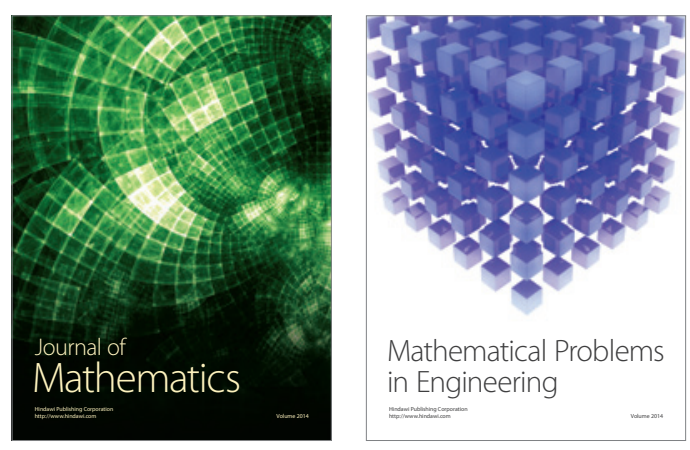

Mathematical Problems in Engineering
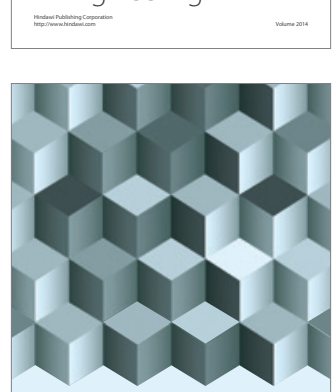

Journal of

Function Spaces
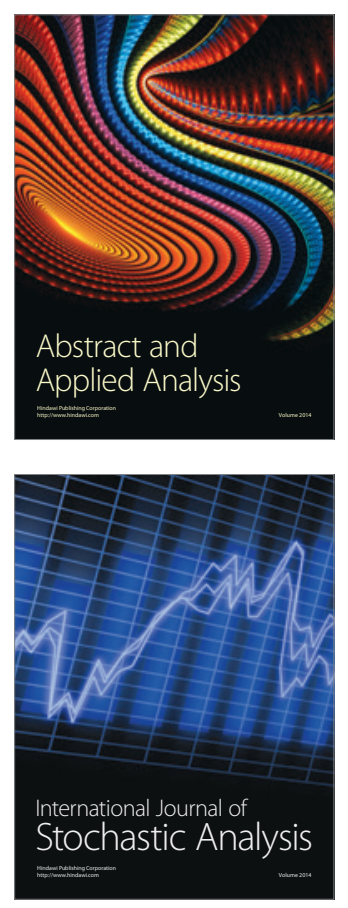

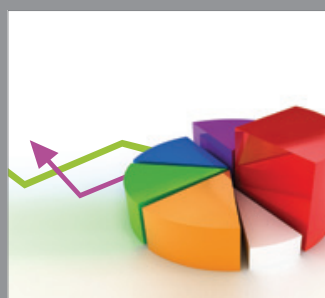

ournal of

Probability and Statistics

Promensencen
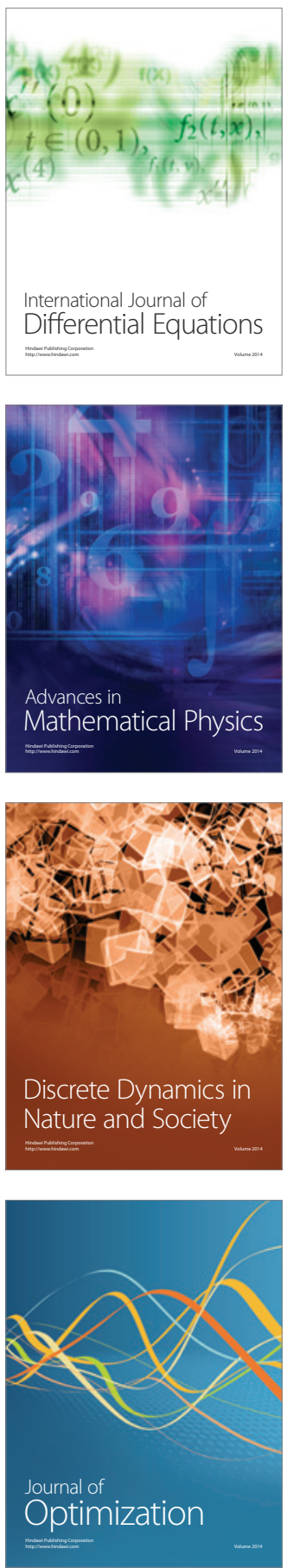